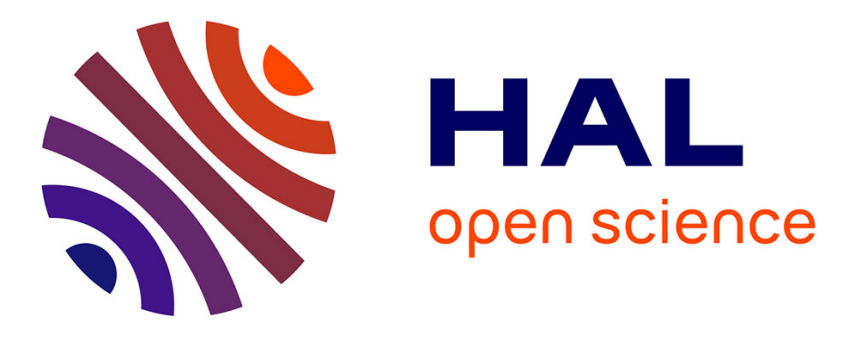

\title{
Mechanism of transmembrane signaling by sensor histidine kinases
}

\author{
Ivan Yu. Gushchin, Igor I Melkinov, Vitaly Polovinkin, Andrii Ishchenko, \\ Anastasia Yuzhakova, Pavel Buslaev, Gleb Bourenkov, Sergei Grudinin, \\ Ekaterina Round, Taras Balandin, et al.
}

\section{To cite this version:}

Ivan Yu. Gushchin, Igor I Melkinov, Vitaly Polovinkin, Andrii Ishchenko, Anastasia Yuzhakova, et al. Mechanism of transmembrane signaling by sensor histidine kinases. Science, 2017, 356 (6342), pp.eaah6345. 10.1126/science.aah6345 . hal-01526454

\section{HAL Id: hal-01526454 https://hal.science/hal-01526454}

Submitted on 11 Jun 2017

HAL is a multi-disciplinary open access archive for the deposit and dissemination of scientific research documents, whether they are published or not. The documents may come from teaching and research institutions in France or abroad, or from public or private research centers.
L'archive ouverte pluridisciplinaire HAL, est destinée au dépôt et à la diffusion de documents scientifiques de niveau recherche, publiés ou non, émanant des établissements d'enseignement et de recherche français ou étrangers, des laboratoires publics ou privés.

$$
\text { Copyright }
$$




\section{Title: Mechanism of transmembrane signaling by sensor histidine kinases}

Authors: Ivan Gushchin ${ }^{1,2, *}$, Igor Melnikov ${ }^{3}$, Vitaly Polovinkin ${ }^{1,2,4}$, Andrii Ishchenko ${ }^{1,5, \# \text {, }}$ Anastasia Yuzhakova ${ }^{1,2}$, Pavel Buslaev ${ }^{2}$, Gleb Bourenkov ${ }^{6}$, Sergei Grudinin ${ }^{7,8,9}$, Ekaterina Round $^{1,4}$, Taras Balandin ${ }^{1}$, Valentin Borshchevskiy ${ }^{1,2}$, Dieter Willbold ${ }^{1,10}$, Gordon Leonard ${ }^{3}$, Georg Büldt ${ }^{2}$, Alexander Popov ${ }^{3}$, Valentin Gordeliy ${ }^{1,2,4, *}$

\section{Affiliations:}

${ }^{1}$ Institute of Complex Systems (ICS), ICS-6: Structural Biochemistry, Research Centre Jülich, 52425 Jülich, Germany.

${ }^{2}$ Moscow Institute of Physics and Technology, 141700 Dolgoprudniy, Russia.

${ }^{3}$ European Synchrotron Radiation Facility, F-38043 Grenoble, France.

${ }^{4}$ Institut de Biologie Structurale J.-P. Ebel, Université Grenoble Alpes-CEA-CNRS, F-38000 Grenoble, France.

${ }^{5}$ Institute of Crystallography, University of Aachen (RWTH), 52056 Aachen, Germany.

${ }^{6}$ European Molecular Biology Laboratory, Hamburg Outstation, 22607 Hamburg, Germany. ${ }^{7}$ Université Grenoble Alpes, LJK, F-38000 Grenoble, France.

${ }^{8}$ CNRS, LJK, F-38000 Grenoble, France.

${ }^{9}$ Inria, F-38000 Grenoble, France.

${ }^{10}$ Institute of Physical Biology, Heinrich-Heine University, 40225 Düsseldorf, Germany

*Correspondence to: I.G. (ivan.gushchin@phystech.edu) and V.G. (valentin.gordeliy@ibs.fr).

${ }^{\#}$ Current address: Department of Chemistry, Bridge Institute, University of Southern California, Los Angeles, CA 90089, USA.

This is the author's version of the work. It is posted here by permission of the AAAS for personal use, not for redistribution. The definitive version was published in Science Vol. 356, Issue 6342, eaah6345, 09 Jun 2017, doi: 10.1126/science.aah6345. 
1 Abstract: One of the major and essential classes of transmembrane (TM) receptors, present in all 2 domains of life, is sensor histidine kinases (HKs), parts of two-component signaling systems 3 (TCS). The structural mechanisms of transmembrane signaling by these sensors are poorly 4 understood. We present here crystal structures of the periplasmic sensor domain, the TM domain 5 and the cytoplasmic HAMP domain of the Escherichia coli nitrate/nitrite sensor HK NarQ in the 6 ligand-bound and mutated ligand-free states. The structures reveal that the ligand binding 7 induces significant rearrangements and piston-like shifts of TM helices. The HAMP domain 8 protomers undergo lever-like motions and convert the piston-like motions into helical rotations. 9 Our findings provide the structural framework for complete understanding of TM TCS signaling 10 and for development of antimicrobial treatments targeting TCS.

11

12 One Sentence Summary: Crystal structures of bacterial nitrate sensor NarQ with and without its 13 ligand reveal a structural basis for signal propagation through the cell membrane in histidine 14 kinases: piston-like motions of transmembrane helices and scissor-like motions in the ubiquitous 15 signaling module HAMP domain. 


\section{Main Text:}

\section{Introduction}

Microorganisms obtain most of the information about their environments through membraneassociated signaling systems. One of the major and most abundant classes of membrane receptors, present in all domains of life, is sensor histidine kinases, members of two-component signaling systems (TCS) (1-3). Overall, tens of thousands of TCS are known (4). Many of these systems are essential for cell growth, survival or pathogenicity and consequently can be targeted to reduce virulence $(5-7)$.

Membrane-associated sensor kinases generally function as homodimers and possess a periplasmic, membrane or intracellular sensor module, a transmembrane (TM) domain, often one or several intracellular signal transduction domains such as HAMP, PAS or GAF, and an intracellular autokinase module (DHp and CA domains), which phosphorylates the response regulator protein $(1,8,9)$ (Figure S1). Other TCS TM sensors, chemo- and photo-receptors, have domain organization similar to that of sensor histidine kinases as they possess the sensor module, TM and HAMP domains, but lack the autokinase module and control separate kinase protein (CheA) via the kinase control module (Figure S1). Also, the core functional unit of chemoreceptors and phototaxis systems is not a dimer but a trimer of dimers (10-12), which may then pack into higher-order oligomeric assemblies (13).

Molecular mechanisms of the TM signal transduction in different classes of TCS are expected to be somewhat similar, since in a vast majority of the sensors there is a dimeric four-helical TM core in the TM domain, often followed by the HAMP domain downstream. At present, only two reports on atomic-resolution structures of the TM domains are available, both describing isolated TM fragments and thus providing little insight into the signal transduction in and out of the TM domain of a typical TCS sensor. The first report provided crystallographic structure of the complex between the photoreceptor sensory rhodopsin and the transmembrane domain of its transducer protein (14). In the second report, NMR structures of non-physiological monomeric TM domains of histidine kinases from three different classes have been described (15). Finally, several low resolution models of the TM helices in the context of full-length proteins such as chemoreceptor Tar or histidine kinase PhoQ have been constructed based on disulfide crosslinking and modeling $(16,17)$. Due to limitations of all these studies, atomic details of TM signal transduction are still not resolved $(8,9)$. As for the HAMP domain, multiple experimental structures of the domains from different proteins are available, and there are several competing hypotheses on the HAMP domain signaling $(8,9,18)$.

To complement the above reports we present here our studies of the Escherichia coli nitrate/nitrite sensor kinase NarQ. Similarly to NarX, another E. coli nitrate/nitrite sensor, NarQ consists of seven major structural elements: the periplasmic sensor domain, the TM domain, the HAMP domain, the signaling helix, the GAF-like domain, the DHp domain and the kinase CA domain (18-20) (Figure S1). While no structural information is available for NarQ, crystal structures of NarX sensor domain in apo and holo states have been determined (21), as well as multiple structures of the HAMP, GAF, DHp and kinase domains of other proteins $(8,9)$. 
1 Here, we used the in meso (22-24) and single wavelength anomalous dispersion (SAD) (25) 2 approaches to crystallize and determine the crystal structures, at resolutions of up to $1.9 \AA$, of a 3 fragment of the nitrate sensor NarQ that contains the sensor, TM and HAMP domains. We 4 determined the symmetric apo state structure of a conservative mutant and symmetric and highly 5 asymmetric holo-S and holo-A state structures of the wild type protein (Figures 1 and S2). 6 Comparison of the apo and holo structures reveals extensive structural rearrangements and 7 provides a direct demonstration of signal transduction through the TM and HAMP domains, 8 including a piston-like motion of TM helices and a lever-like rotation of HAMP domain 9 protomers. Comparison of holo-S and holo-A structures shows that the signal transduction in 10 NarQ can be achieved via different sets of conformational changes. Overall, the presented 11 findings clarify the mechanism of TM signaling in TCS receptors. 


\section{Results}

Structure of the sensor domain

In all of the presented structures, including the asymmetric ligand-bound form, the NarQ sensor domain is a symmetric dimer of two monomers comprised of four $\alpha$-helices $\mathrm{H} 1-\mathrm{H} 4$, with the helices $\mathrm{H} 2$ and $\mathrm{H} 4$ being broken into sub-helices (Figure S3). The loop between the helices $\mathrm{H} 2$ and $\mathrm{H} 3$ is anchored into the membrane by residues Trp 89 and Tyr90. Nitrate binds at the symmetry axis between the helices $\mathrm{H} 1$ and $\mathrm{H} 1$ ' of the two protomers, where it is coordinated by the side chains of the residues Arg50 and Arg50' (Figure S3E). The ligand was observed in all crystals of the wild type (WT) protein, including those obtained without supplementation of the protein purification and crystallization solutions with nitrate. This is probably a consequence of the combination of following factors: high ligand affinity of the crystallized fragment for nitrate, presence of nitrate impurities in the aforementioned solutions and the very low amount of nitrate needed to saturate the binding sites in the $\sim 0.5$ milimolar receptor solution used for crystallization. Consequently, we generated a conservative R50K mutant to obtain the crystal structure of NarQ in its apo state. This mutation is known to lock the receptor in the off state (26) but was not expected to alter the local structure of the sensor domain since arginine and lysine side chains are similar in size and charge. Indeed, the structures of the NarQ sensor domain in the apo R50K and holo-S and holo-A WT forms reported here closely resemble those of the apo and holo WT NarX sensor domain (21), respectively. In particular, the positions of the membrane-proximal ends of helices $\mathrm{H} 1$ and $\mathrm{H} 4$, responsible for downstream signaling, are identical in the corresponding structures (Figures S3C,F) and we thus conclude that introduction of the mutation results in correct reproduction of the structural features of the WT ligand-free protein.

\section{Structure of the TM domain}

In all of the structures presented here, the NarQ TM domain is an anti-parallel 4-stranded coiled coil (CC) consisting of 9 CC layers (Figures 1, 2 and S4). Several ordered lipid fragments are observed in the holo-S structure, one fragment in observed in the holo-A structure, and no fragments are observed in the apo structure. However, due to the small size of the fragments, reliable identification of the corresponding lipidic moieties is not possible.

There are notable variations in the packing of the TM helices. The apo state structure is best described as a dimeric CC comprised of helices TM1 and TM1', with TM2 and TM2' flanking this. The symmetric holo-S state structure is a traditional four-helical CC, switching from a 7/2period left-handed $\mathrm{CC}$ at the periplasmic side to a 11/3-period structure with close to zero supercoil twist on the cytoplasmic side. Finally, in the asymmetric holo-A state, the TM domain is a highly distorted CC, where the relative arrangement of the helices in layers 1-4 and 9 resembles that observed in the symmetric holo-S state and the arrangement in layers 7 and 8 resembles that seen in the apo state (Figure S4).

In general, the cytoplasm-proximal layers are more hydrophobic and are packed tightly, whereas those proximal to the periplasm contain hydrated cavities and multiple polar (Ser22, Ser25, Thr26, Thr32, Ser154, Thr163) and somewhat disordered glycine residues (Gly27, Gly157, 
Gly158 and Gly160, Figure S2E). While the role of water molecules and glycine residues in the NarQ TM domain is not clear, similar $\alpha$-helix-destabilizing features are observed in the TM domains of other histidine kinases (Figure S5) and they probably impart the TM domain with the flexibility needed to accommodate signaling-associated transitions, similarly to the glycine hinge in the cytoplasmic part of chemoreceptors (27).

The $\alpha$-helical composition of the TM region of TCS receptors has long been predicted $(8,9,14$ 17). However, the structure of the NarQ TM domain is notably different from that of the sensory rhodopsin transducer TM domain (Figure S5). In NarQ, the TM region is either a simple 4helical CC, or has a dimeric CC core consisting of the helices TM1, whereas $N p H$ trII has a dimeric CC core consisting of the helices TM2 $(14,28)$. Also, in NarQ, there is either zero or left-handed supercoil twist, while in $\mathrm{NpHtrII}$ this changes from close to zero at the periplasmic side to right-handed at the cytoplasmic side (Figure S5). As for the comparison with the TM fragments of $\mathrm{ArcB}, \mathrm{QseC}$ and $\mathrm{KdpD}$, unfortunately, the NMR structures do not provide the information about the dimerization interfaces and $\mathrm{CC}$ packing in the physiological dimers of any of these kinases (15).

\section{The junctions between TM and adjacent domains.}

Our crystal structures of NarQ reveal details of the interactions of the TM domain with the membrane-proximal domains (Figure S6). On the periplasmic side of the protein, in the apo state, there is a continuous transition of the helices TM1 and TM2 into the helices H1 and H4, respectively, whereas in both holo-A and holo-S state there is a break in the $\alpha$-helical structure between TM1 and H1 (see Figures S6A,D for comparison of apo and holo-S states).

At the cytoplasmic side, in all states the cytoplasmic side of TM1 and the protein's N-terminus make extensive contacts with the HAMP domain. The highly conserved glutamic acid residue Glu207 of the membrane-proximal region of HAMP domain helix AS2 (29) forms two hydrogen bonds with the backbone nitrogens of helix TM1 residues (Figures S6B,E), similarly to what has been observed in the structure of the soluble mutant of protein Af1503 (30) (Figure S7A). As for the TM2-AS1 junction, there is a proline-induced kink (Figures S6C,F), again as also seen for the protein Af1503 (30) (Figure S7B).

\section{The HAMP domain}

The NarQ HAMP domain displays a typical parallel 4-stranded CC fold (18), with each protomer consisting of two $\alpha$-helices AS1 and AS2, separated by an unstructured linker (Figure S8). In all of the structures, the HAMP domain is less ordered than the sensor and TM domains, and in the apo state structure, the positions of the side chains of Leu225 and Tyr226 are not resolved. Overall, the apo state structure is similar to the structure of the Af1503 HAMP domain (Figure S9). The structure observed for the symmetric holo-S state HAMP domain is influenced by crystal contacts: the helices AS2 are interspersed with the same helices of an adjacent dimer (Figures S2 and S8), and consequently there is no contact between the Leu225 side chains facing towards the CC core. While such conformation would be consistent with an antiparallel 4helical CC downstream domain, such as in bacterial chemoreceptors (31), it is unusual in the structural context of histidine kinases. The crystal contacts mentioned above are absent in the 
asymmetric holo-A state structure (space group P2). There, the hydrophobic Leu225 side chains are less exposed to the solvent and are in contact with each other. Thus, we conclude that the holo-A structure represents a better model of the activated NarQ HAMP domain.

\section{The signaling helix region}

In order to obtain insights into signal transduction through and downstream of the HAMP domain, we have constructed atomic models of the adjacent signaling helix region (residues 224245, see NarQ domain architecture in Figure S1). The signaling helix is a common sequence motif in sensor kinases (32) and sensory rhodopsin transducers (33), and two CC heptad repeat pattern assignments are possible for NarQ based on the positions of hydrophobic and hydrophilic residues (Figure S10). Neither of the models is compatible with the holo state HAMP domain structures, but both, especially model 2 , are compatible with the structure of the apo state HAMP domain (see layer 6 in Figure S8 and layer 1 in Figure S10). Model 1 comprises a phase stutter that is also observed in other histidine kinases and chemoreceptors $(18,19,34)$ and may be required for destabilization of the signaling helix for it to be able to adopt different signaling states. However, model 2 appears to be more probable judging from the protein sequence (19).

\section{Discussion}

\section{Signal transduction in NarQ}

Comparison of the apo and holo state crystal structures of NarQ reveals that binding of nitrate causes significant rearrangements in the TM region that can be represented as a combination of changes in the lateral arrangement of the TM helices and piston-like shifts of the helices in the direction perpendicular to the membrane plain. At the same time, comparison of the holo-S and holo-A states reveals profound differences in the middle of the TM segment but identical conformations at its periplasmic and intracellular sides (see Movie S1 for comparison of apo and holo-S state structures, and Movie S2 for comparison of holo-S and holo-A structures). We make several observations from these comparisons.

First, binding of nitrate in the vicinity of Gly47 causes disruption in the $\alpha$-helix H1 and appearance of a 310 -helix element: Gly47 carbonyl switches from Met51 amide hydrogen to Arg50 amide hydrogen (from $i \rightarrow i+4$ helical hydrogen bonding to $i \rightarrow i+3$, Figures S3 and S11). $3_{10}$-helices have a higher helical rise per residue than $\alpha$-helices (35), and as a result the membrane-proximal part of $\mathrm{H1}$ moves slightly towards the membrane and rotates. This rotation of the membrane-proximal part of H1 opens a "hole" near the residues Ile43 and Ala46, which is then occupied by Val136 "knob" (36). Consequently, H4 rotates as well and moves away from the membrane, which results in a piston-like displacement of $\mathrm{H} 4$ relative to $\mathrm{H} 1$ for $~ 0.5-1.0 \AA$. Also, the helices $\mathrm{H} 4$ and $\mathrm{H}^{\prime}$ ' are brought much closer to the helices $\mathrm{H} 1$ and H1'. This interpretation is supported by mutagenesis data: mutation of Ala46 to serine is inconsequential, mutation to threonine results in impaired signaling, and mutations to much bulkier asparagine and isoleucine lock NarQ in the 'off' state (37). The observed conformational changes in NarQ are identical to the changes observed in isolated NarX sensor domain (Figure S3) (21). 
Rotation of the membrane-proximal parts of $\mathrm{H} 1$ and $\mathrm{H} 1^{\prime}$ and approach of $\mathrm{H} 4$ and $\mathrm{H} 4$ ' upon binding of nitrate result in disruption of the TM1/H1-TM1'/H1' $\mathrm{CC}$ interface, appearance of discontinuity between the $\alpha$-helices TM1 and H1, and distancing of TM1 and TM1' periplasmic sides (Figures 3 and S11, and Movie S1). The introduced discontinuity between TM1 and H1 further increases the relative piston-like displacement from $\sim 1 \AA$ for $\mathrm{H} 1$ relative to $\mathrm{H} 4$ (as in NarX, (21)) to $\sim 2.5 \AA$ for TM1 relative to TM2 (Figure S11). Importance of the TM1-H1 helical break for kinase activation is also highlighted by mutagenesis study: introduction of a proline into the TM1-H1 junction (mutation E41P) locks the receptor in the 'on' state, whereas other nonconservative mutations of Glu41 (E41R, E41H and E41L) do not disrupt the function of the receptor (37).

Whereas conformational changes in the sensor domain and at the sensor-TM junction are moderate and identical in holo-A and holo-S structures, the rearrangements in the TM domain are much more pronounced and are different between holo-A and holo-S. Helices TM1, which form a tight CC interface in the apo state, are not fully separated in the holo-A structure (Figure S4). In the holo-S state the four TM helices form a traditional four-helical coiled coil (Figures 2 and S4). However, there is a similarity between the holo-A and holo-S structures: in both the $\sim 2.5 \AA$ relative piston-like displacement of the TM helices is fully transduced towards the HAMP domain (Figures 2 and 3, Movies S1 and S2). Conformational changes at the TMHAMP interface are also identical in the holo-S and holo-A structures as compared to the apo state structure. Thus, comparison of the asymmetric holo-A and symmetric holo-S state structures presents an insight into receptor flexibility and robustness of signal transduction by the TM domain despite the $\sim 9 \AA$ difference in the position of the HAMP domain (Figures 1, 3, S4 and S8).

Finally, in the cytoplasmic part of NarQ, the piston-like displacements of the TM helices, caused by binding of the ligand, are amplified through the lever-like rotations of the HAMP domain protomers around the Pro179 hinge into roughly $7 \AA$ displacements of the output ends of the AS2 helices (Figure 3). During these conformational rearrangements, each HAMP domain protomer behaves essentially as a rigid body. RMSD of the backbone atoms positions of the helices AS1 (residues 179 to 192) and AS2 (residues 206 to 225) is $\sim 0.6 \AA$ between the apo and both holo-S and holo-A structures, and $\sim 0.4 \AA$ between the holo-S and holo-A structures, which is comparable to crystal-to-crystal variation and typical crystallographic error of atomic positions. However, due to relative motions of the HAMP domain protomers, the whole dimeric HAMP domain itself changes significantly: RMSD of the backbone atoms positions of the helices AS1 (residues 179 to 192) and AS2 (residues 206 to 225) is $\sim 2.9 \AA$ between the apo and holo-S structures and $3.0 \AA$ between the apo and holo-A structures, as opposed to $\sim 1.2 \AA$ between the holo-S and holo-A structures.

Details of the signal transduction in NarQ downstream of the HAMP domain are less clear due to truncation of the crystallized construct. In the apo state, the CC register of the AS2 output ends is consistent with the CC register of the signaling helix. However, in the holo-A and holo-S states the $\mathrm{CC}$ helical phase is much less consistent with that of the signaling helix. Therefore, it appears that the signaling helix should be destabilized and/or dissociated upon binding of the ligand (Figure 4). Signaling-related dissociation or destabilization of signaling helix was also observed 
in Af1503-EnvZ chimeras (34) and histidine kinases BvgS and DesK $(38,39)$ and may thus be the general mechanism for signal propagation towards the DHp domain in TCS sensor kinases.

\section{Mechanism of transmembrane signaling by TCS receptors}

Several hypotheses concerning transmembrane signaling mechanisms in TCS receptors have been proposed $(8,9)$. For sensor histidine kinases, the crystal structures of $\alpha$-helical NarX and TorS sensor domains hinted at piston-like conformational changes $(21,40)$. The crystal structures of the mixed $\alpha / \beta$ tandem PAS sensor domain of HAMP-less kinase LuxQ revealed rotation of signal output helices (41), which was also demonstrated in another HAMP-less kinase AgrC where the sensor domain is integral to the membrane (42). Recent crosslinking study revealed scissor-like diagonal displacement of the TM helices in the kinase PhoQ (17). For chemoreceptors with $\alpha$-helical sensor domains, such as aspartate receptor Tar, the major model is piston-like displacement of the TM helices in the direction normal to the membrane plane (13, $31)$. For chemoreceptor McpB with mixed $\alpha / \beta$ tandem PAS sensor domain, the signal was found to be transduced via rotation of TM helices (43). Finally, in the sensory rhodopsin-cognate transducer complex, where sensor is integral to the membrane, a combination of a piston-like motion and a rotation of the signal output helices was observed (44). While the piston-like displacements, helical rotations, $\mathrm{CC}$ phase changes, and scissoring motions are not mutually exclusive and possibly coupled to each other, the consensus on the transmembrane signaling mechanism in TCS sensors is currently lacking $(8,9)$.

Our results show that binding of ligand to NarQ causes a piston-like displacement of the TM helices, which is accompanied by extensive symmetric or asymmetric rearrangements and scissoring of the TM helices. The rearrangements are different in the two presented holo state structures, but the piston-like displacement is perfectly conserved. Thus, the latter appears to be a more robust mechanism of TM signal transduction.

Also, our data shows that symmetrical changes in the sensor domain may result both in symmetrical changes in the TM domain (as in the holo-S structure), and in asymmetrical changes (as in the holo-A structure). Asymmetry only changes the position of the HAMP domain relative to the TM domain and not its signaling state (Figure 1). Sensor domains of several histidine kinases are known to reside in asymmetric conformations in the inactive state and in symmetric conformations in the active state (40). The aspartate chemoreceptor can be activated both by asymmetric changes in the sensor domain upon binding of a ligand $(31,45)$ and presumably symmetric conformational changes upon mutations of aminoacids at the membrane/water interfaces $(46,47)$. Thus, whether the asymmetric changes in the sensor domain can result in symmetric changes in the TM domain, and the exact relations between asymmetry and activation, are not clear and remain to be investigated.

\section{Signal transduction through the HAMP domain}

Currently, there are several competing hypotheses concerning the mechanism of signal transduction through the HAMP domain $(8,9,18)$ : the gearbox model postulates rotation of the HAMP domain helices in opposite directions $(48,49)$; the dynamic bundle model proposes that signal transduction is associated with changes in HAMP domain stability $(50,51)$; several 
reports based on crosslinking and crystallographic studies propose a variety of scissoring models $(52-54)$.

Our results indicate that in NarQ the HAMP domain serves as an amplifier and convertor of the piston-like conformational changes in the TM domain. Piston-like displacements of TM1 and TM1' cytoplasmic ends for $2.5 \AA$ relative to the proline hinge result in lever-like rotations of the protomers around their respective proline hinges and consequent $7 \AA$ displacements in opposite directions of AS2 and AS2' membrane-distal ends. This results in a $\sim 90^{\circ}$ change in the helical phase and the output CC register (Figures 3 and S8). Thus, the membrane-proximal HAMP domain acts as a convertor of the piston-like motions of TM domain helices into helical rotation at its output helices. These conformational changes are similar to the scissoring models (52-54), and are possibly accompanied by changes in the stability of the HAMP or adjacent domains. However, our results do not support the gearbox hypothesis. We observe that the HAMP domain protomers move relative to each other in a rigid body fashion, without rotation of the helices AS1 and AS2 within each protomer. Also, we should note that observation of slightly different HAMP domain conformations in holo-A and holo-S structures and increased B-factors in the HAMP domain region underline its dynamic nature. It is possible that the domain exists in an ensemble of similar conformations in vivo, whereas crystallographic structures provide only static snapshots. Finally, due to absence of the adjacent signaling helix region in the crystallized construct, the observed conformational changes may under- or overestimate the actual transformations associated with signal transduction.

Interestingly, signal convertor role of the HAMP domain might reconcile the data on TM signal transduction in histidine kinases that contain and those that lack the HAMP domains. In sensors containing a membrane-proximal HAMP domain, binding of a ligand causes a piston-like displacement of the TM helices (that may be accompanied by scissoring), which is then converted into helical rotation by the HAMP domain. In sensors lacking the HAMP domain, binding of ligand directly causes rotation of the TM signal output helices $(41,42)$. In both cases the signal that is passed downstream is the helical rotation. Whether piston-like displacements are present during PhoQ activation remains to be tested.

\section{Conclusions}

In this work, we described the structures of the TM and membrane-proximal domains of the nitrate/nitrite receptor NarQ, and the conformational changes induced upon the binding of nitrate. The multiple conformational states observed for the TM domains present a template for studies of other TCS receptors, establish the importance of the piston-like displacements of the TM helices and highlight the role of the HAMP domain as an amplifier and convertor of a piston-like displacement into helical rotation. Overall, the results show how a mechanistic signal is generated and amplified while being transduced through the protein over distances of $100 \AA$ or more. Since membrane-associated TCS are ubiquitous in microorganisms and are central for bacterial sensing (1-4), we believe that the results reported here will help in understanding of a broad range of cellular processes, ranging from basic metabolism to sporulation, quorum sensing and virulence. They may also provide insights for development of novel antimicrobial treatments targeting TCS (5-7). 
Materials and Methods:

\section{Cloning, protein expression and purification}

The nucleotide sequence encoding residues 1-230 of NarQ was cloned from Escherichia coli strain BL21 (DE3) and introduced into the pSCodon1.2 expression vector (StabyCodon T7, Eurogentec, Belgium) via NdeI and XhoI restriction sites. Consequently, the construct harbored C-terminal $6 \times$ His tag. Mutation R50K was introduced by site-directed mutagenesis. Both the WT protein and the mutant version were expressed in E. coli strain SE1 (StabyCodon T7, Eurogentec, Belgium). Cells were cultured in shaking baffled flasks in ZYP-5052 auto-inducing medium (55) containing $100 \mathrm{mg} / 1$ ampicillin. After 2 hours of incubation at $37{ }^{\circ} \mathrm{C}$, the temperature was decreased to $30{ }^{\circ} \mathrm{C}$ and incubation continued overnight. Harvested cells were disrupted in M-110P Lab Homogenizer (Microfluidics, USA) at 25000 psi in a PBS buffer with addition of $50 \mathrm{mg} / 1$ DNase I (Sigma-Aldrich, USA) and EDTA-free protease inhibitor cocktail Complete (Roche). The membrane fraction of cell lysate was isolated by ultracentrifugation at $90000 \mathrm{~g}$ for $1 \mathrm{~h}$ at $4^{\circ} \mathrm{C}$. The pellet was resuspended in a buffer containing $50 \mathrm{mM}$ $\mathrm{NaH}_{2} \mathrm{PO}_{4} / \mathrm{Na}_{2} \mathrm{HPO}_{4}, \mathrm{pH} 8.0,0.3 \mathrm{M} \mathrm{NaCl}$ and $2 \%$ DDM (Anatrace, Affymetrix, USA) and stirred overnight for solubilization. The insoluble fraction was then removed by ultracentrifugation at $90000 \mathrm{~g}$ for $1 \mathrm{~h}$ at $4^{\circ} \mathrm{C}$. The supernatant was loaded on a gravity flow column containing NiNTA resin (Qiagen, Germany) and the protein was eluted in a buffer containing $20 \mathrm{mM}$ Tris, $\mathrm{pH}$ 8.0, $0.3 \mathrm{M} \mathrm{NaCl}, 0.2 \mathrm{M}$ imidazole and 0.1\% DDM. Imidazole was removed by dialysis against $20 \mathrm{mM}$ Tris, $\mathrm{pH}$ 8.0, $0.3 \mathrm{M} \mathrm{NaCl}$ and $0.1 \%$ DDM for 3 hours. The eluate was subjected to sizeexclusion chromatography on $125 \mathrm{ml}$ Superdex 200 PG column (GE Healthcare Life Sciences, USA) in a buffer containing $20 \mathrm{mM}$ Tris, $\mathrm{pH} 8.0,0.3 \mathrm{M} \mathrm{NaCl}, 0.1 \%$ DDM. Protein-containing fractions were pooled and concentrated to $30 \mathrm{mg} / \mathrm{ml}$ for crystallization.

\section{Crystallization}

The crystals were grown using the in meso approach (22-24), similarly to our previous work (14, $56,57)$. The solubilized protein in the crystallization buffer was added to the monooleoyl-formed lipidic phase (Nu-Chek Prep, USA). Crystallization trials were set up using the NT8 robotic system (Formulatrix, USA). The crystals were grown at $22{ }^{\circ} \mathrm{C}$ and reached the final size of $50-$ $150 \mu \mathrm{m}$ within 2 weeks. The best holo state crystals in space groups F222 and P2 were obtained using the precipitants $0.6 \mathrm{M} \quad \mathrm{KH}_{2} \mathrm{PO}_{4} / \mathrm{Na}_{2} \mathrm{HPO}_{4} \mathrm{pH}$ 4.6, $5 \mathrm{mM} \mathrm{NaNO}_{3}$ and $0.3 \mathrm{M}$ $\mathrm{KH}_{2} \mathrm{PO}_{4} / \mathrm{Na}_{2} \mathrm{HPO}_{4} \mathrm{pH} 7.6,250 \mathrm{mM} \mathrm{NaBr}$, respectively. The best apo state crystals in the space group I2 $2_{1} 2_{1} 2_{1}$ were obtained using the precipitant $1.6 \mathrm{M}\left(\mathrm{NH}_{4}\right)_{3} \mathrm{PO}_{4} \mathrm{pH}$ 5.8. Before harvesting, the crystals were incubated for $5 \mathrm{~min}$ in the respective precipitant solutions supplemented with $20 \%$ glycerol. For iodide-SAD experiments, the soaking solution additionally contained $0.5 \mathrm{M}$ NaI. All crystals were harvested using micromounts (MiTeGen), and were flash-cooled and stored in liquid nitrogen.

\section{Acquisition and treatment of diffraction data}

The diffraction data were collected at $100 \mathrm{~K}$ at ESRF beamline ID23-1 (58) equipped with a PILATUS 6M-F detector. The data collection statistics are reported in Table S1. In all cases the diffraction was anisotropic as determined by decay of the $\mathrm{CC}_{1 / 2}$ values in $20^{\circ}$ cones along the 
reciprocal cell directions $\mathrm{a}^{*}, \mathrm{~b}^{*}$, and $\mathrm{c}^{*}(59)$. In the space group $\mathrm{I} 2{ }_{1} 2{ }_{1} 2_{1}$, the resolution limits along the directions $\mathrm{a}^{*}$ and $\mathrm{b}^{*}$ were 2.55 and $2.17 \AA$, and along the direction $\mathrm{c}^{*}$ the $\mathrm{CC}_{1 / 2}$ value was $\sim 0.9$ at the resolution cut-off of $1.9 \AA$. In the native dataset in the space group F222, the resolution limits along the directions $\mathrm{a}^{*}$ and $\mathrm{b}^{*}$ were 2.26 and $1.94 \AA$, and along the direction $\mathrm{c}^{*}$ the $\mathrm{CC}_{1 / 2}$ value was $\sim 0.8$ at the resolution cut-off of $1.94 \AA$. In the space group P2, the resolution limit along the direction $\mathrm{a}^{*}$ was $2.82 \AA$, and along the directions $\mathrm{b}^{*}$ and $\mathrm{c}^{*}$ the $\mathrm{CC}_{1 / 2}$ values were $\sim 0.6$ and 0.9 at the resolution cut-off of $2.42 \AA$. Diffraction images were processed using XDS (60). XSCALE (60) was used to merge different datasets and to scale the data for the phasing steps. POINTLESS (59) and AIMLESS (59) were used to merge, scale, assess the quality, convert intensities to structure factor amplitudes and generate Free-R labels.

\section{Structure determination and refinement}

The holo-S state structure in the space group F222 was solved using experimental phasing, and the apo and holo-A state structures in the space groups $\mathrm{I} 22_{1} 2_{1} 2_{1}$ and $\mathrm{P} 2$, respectively, were solved using molecular replacement with MOLREP (61) and the sensor domain from the structure in the space group F222 as a search model. For the F222 structure solution, 8 isomorphous datasets from NaI-soaked crystals were collected and merged for subsequent SAD phasing. The iodide sites were determined using ShelxD (62) with the HKL2MAP (63) interface, with the $\mathrm{CC}_{\text {all }}$ and $\mathrm{CC}_{\text {weak }}$ values of $40.0 \%$ and $15.4 \%$. The resolution was extended to $1.94 \AA$ using the native F222 data collected from a single crystal. 178 Ala residues were placed in $5 \times 50$ cycles of autotracing and density modification using ShelxE (62). Initial model was improved using automatic model building software ARP-wARP (64). The final F222 model was built using only the native data. For the I $22_{1} 2_{1} 2_{1}$ and P2 structure solution, native datasets collected from single crystals were used in each case. All models were refined manually using Coot (65) and REFMAC5 (66). Intermediate conformations shown in movies S1 and S2 were calculated using the NOLB algorithm (68). 


\section{References and Notes:}

1. T. Krell et al., Bacterial Sensor Kinases: Diversity in the Recognition of Environmental Signals. Annual Review of Microbiology. 64, 539-559 (2010).

2. A. M. Stock, V. L. Robinson, P. N. Goudreau, Two-Component Signal Transduction. Annual Review of Biochemistry. 69, 183-215 (2000).

3. P. M. Wolanin, P. A. Thomason, J. B. Stock, Histidine protein kinases: key signal transducers outside the animal kingdom. Genome Biol. 3, 1-8 (2002).

4. L. E. Ulrich, I. B. Zhulin, The MiST2 database: a comprehensive genomics resource on microbial signal transduction. Nucleic Acids Res. 38, D401-407 (2010).

5. Y. Gotoh et al., Two-component signal transduction as potential drug targets in pathogenic bacteria. Current Opinion in Microbiology. 13, 232-239 (2010).

6. B. LaSarre, M. J. Federle, Exploiting Quorum Sensing To Confuse Bacterial Pathogens. Microbiol. Mol. Biol. Rev. 77, 73-111 (2013).

7. D. A. Rasko, V. Sperandio, Anti-virulence strategies to combat bacteria-mediated disease. Nat Rev Drug Discov. 9, 117-128 (2010).

8. C. P. Zschiedrich, V. Keidel, H. Szurmant, Molecular Mechanisms of Two-Component Signal Transduction. Journal of Molecular Biology. 428, 3752-3775 (2016).

9. M. P. Bhate, K. S. Molnar, M. Goulian, W. F. DeGrado, Signal Transduction in Histidine Kinases: Insights from New Structures. Structure. 23, 981-994 (2015).

10. M. Li, G. L. Hazelbauer, Core unit of chemotaxis signaling complexes. Proceedings of the National Academy of Sciences. 108, 9390-9395 (2011).

11. C. M. Khursigara, X. Wu, S. Subramaniam, Chemoreceptors in Caulobacter crescentus: Trimers of Receptor Dimers in a Partially Ordered Hexagonally Packed Array. J. Bacteriol. 190, 6805-6810 (2008).

12. P. S. Orekhov et al., Signaling and Adaptation Modulate the Dynamics of the Photosensoric Complex of Natronomonas pharaonis. PLOS Computational Biology. 11, e1004561 (2015).

13. G. L. Hazelbauer, J. J. Falke, J. S. Parkinson, Bacterial chemoreceptors: high-performance signaling in networked arrays. Trends in Biochemical Sciences. 33, 9-19 (2008).

14. V. I. Gordeliy et al., Molecular basis of transmembrane signalling by sensory rhodopsin IItransducer complex. Nature. 419, 484-487 (2002).

15. I. Maslennikov et al., Membrane domain structures of three classes of histidine kinase receptors by cell-free expression and rapid NMR analysis. PNAS. 107, 10902-10907 (2010). 
16. A. A. Pakula, M. I. Simon, Determination of transmembrane protein structure by disulfide cross-linking: the Escherichia coli Tar receptor. PNAS. 89, 4144-4148 (1992).

17. K. S. Molnar et al., Cys-Scanning Disulfide Crosslinking and Bayesian Modeling Probe the Transmembrane Signaling Mechanism of the Histidine Kinase, PhoQ. Structure. 22, 12391251 (2014).

18. J. S. Parkinson, Signaling Mechanisms of HAMP Domains in Chemoreceptors and Sensor Kinases. Annual Review of Microbiology. 64, 101-122 (2010).

19. V. Stewart, L.-L. Chen, The S Helix Mediates Signal Transmission as a HAMP Domain Coiled-Coil Extension in the NarX Nitrate Sensor from Escherichia coli K-12. J. Bacteriol. 192, 734-745 (2010).

20. V. Stewart, Nitrate- and nitrite-responsive sensors NarX and NarQ of proteobacteria. Biochemical Society Transactions. 31, 1-10 (2003).

21. J. Cheung, W. A. Hendrickson, Structural Analysis of Ligand Stimulation of the Histidine Kinase NarX. Structure. 17, 190-201 (2009).

22. V. I. Gordeliy, R. Schlesinger, R. Efremov, G. Büldt, J. Heberle, in Membrane Protein Protocols: Expression, Purification, and Characterization (ed. Selinsky, B) (Humana Press, Totowa, New Jersey, 2003).

23. M. Caffrey, Crystallizing Membrane Proteins for Structure Determination: Use of Lipidic Mesophases. Annual Review of Biophysics. 38, 29-51 (2009).

24. V. Cherezov, Lipidic cubic phase technologies for membrane protein structural studies. Current Opinion in Structural Biology. 21, 559-566 (2011).

25. Z. Dauter, M. Dauter, K. R. Rajashankar, Novel approach to phasing proteins: derivatization by short cryo-soaking with halides. Acta Crystallographica Section D Biological Crystallography. 56, 232-237 (2000).

26. S. B. Williams, V. Stewart, Discrimination between structurally related ligands nitrate and nitrite controls autokinase activity of the NarX transmembrane signal transducer of Escherichia coli K-12. Molecular Microbiology. 26, 911-925 (1997).

27. M. D. Coleman, R. B. Bass, R. S. Mehan, J. J. Falke, Conserved Glycine Residues in the Cytoplasmic Domain of the Aspartate Receptor Play Essential Roles in Kinase Coupling and On-Off Switching †. Biochemistry. 44, 7687-7695 (2005).

28. A. Ishchenko et al., Ground state structure of D75N mutant of sensory rhodopsin II in complex with its cognate transducer. Journal of Photochemistry and Photobiology B: Biology. 123, 55-58 (2013). 
29. S. Dunin-Horkawicz, A. N. Lupas, Comprehensive Analysis of HAMP Domains: Implications for Transmembrane Signal Transduction. Journal of Molecular Biology. 397, 1156-1174 (2010).

30. M. D. Hartmann et al., A soluble mutant of the transmembrane receptor Af1503 features strong changes in coiled-coil periodicity. Journal of Structural Biology. 186, 357-366 (2014).

31. J. S. Parkinson, G. L. Hazelbauer, J. J. Falke, Signaling and sensory adaptation in Escherichia coli chemoreceptors: 2015 update. Trends in Microbiology. 23, 257-266 (2015).

32. V. Anantharaman, S. Balaji, L. Aravind, The signaling helix: a common functional theme in diverse signaling proteins. Biology Direct. 1, 25 (2006).

33. I. Gushchin, V. I. Gordeliy, S. Grudinin, Role of the HAMP Domain Region of Sensory Rhodopsin Transducers in Signal Transduction. Biochemistry. 50, 574-580 (2011).

34. H. U. Ferris et al., Mechanism of Regulation of Receptor Histidine Kinases. Structure. 20, 56-66 (2012).

35. R. S. Vieira-Pires, J. H. Morais-Cabral, 310 helices in channels and other membrane proteins. J Gen Physiol. 136, 585-592 (2010).

36. F. H. Crick, The packing of $\alpha$-helices: simple coiled-coils. Acta crystallographica. 6, 689697 (1953).

37. R. C. Chiang, R. Cavicchioli, R. P. Gunsalus, "Locked-on" and "locked-off" signal transduction mutations in the periplasmic domain of the Escherichia coli NarQ and NarX sensors affect nitrate- and nitrite-dependent regulation by NarL and NarP. Mol. Microbiol. 24, 1049-1060 (1997).

38. E. Lesne et al., Balance between Coiled-Coil Stability and Dynamics Regulates Activity of BvgS Sensor Kinase in Bordetella. mBio. 7, e02089-15 (2016).

39. E. Saita et al., A coiled coil switch mediates cold sensing by the thermosensory protein DesK. Molecular Microbiology. 98, 258-271 (2015).

40. J. O. Moore, W. A. Hendrickson, An Asymmetry-to-Symmetry Switch in Signal Transmission by the Histidine Kinase Receptor for TMAO. Structure. 20, 729-741 (2012).

41. M. B. Neiditch et al., Ligand-Induced Asymmetry in Histidine Sensor Kinase Complex Regulates Quorum Sensing. Cell. 126, 1095-1108 (2006).

42. B. Wang, A. Zhao, R. P. Novick, T. W. Muir, Activation and Inhibition of the Receptor Histidine Kinase AgrC Occurs through Opposite Helical Transduction Motions. Molecular Cell. 53, 929-940 (2014). 
43. H. Szurmant, M. W. Bunn, S. H. Cho, G. W. Ordal, Ligand-induced Conformational Changes in the Bacillus subtilis Chemoreceptor McpB Determined by Disulfide Crosslinking in vivo. Journal of Molecular Biology. 344, 919-928 (2004).

44. R. Moukhametzianov et al., Development of the signal in sensory rhodopsin and its transfer to the cognate transducer. Nature. 440, 115-119 (2006).

45. S. A. Chervitz, J. J. Falke, Molecular mechanism of transmembrane signaling by the aspartate receptor: a model. Proceedings of the National Academy of Sciences of the United States of America. 93, 2545-2550 (1996).

46. A. S. Miller, J. J. Falke, Side Chains at the Membrane-Water Interface Modulate the Signaling State of a Transmembrane Receptor. Biochemistry. 43, 1763-1770 (2004).

47. R. R. Draheim, A. F. Bormans, R. Lai, M. D. Manson, Tryptophan Residues Flanking the Second Transmembrane Helix (TM2) Set the Signaling State of the Tar Chemoreceptor. Biochemistry. 44, 1268-1277 (2005).

48. M. Hulko et al., The HAMP Domain Structure Implies Helix Rotation in Transmembrane Signaling. Cell. 126, 929-940 (2006).

49. H. U. Ferris et al., The Mechanisms of HAMP-Mediated Signaling in Transmembrane Receptors. Structure. 19, 378-385 (2011).

50. Q. Zhou, P. Ames, J. S. Parkinson, Mutational Analyses of HAMP Helices Suggest a Dynamic Bundle Model of Input-Output Signaling in Chemoreceptors. Mol Microbiol. 73, 801-814 (2009).

51. V. Stewart, The HAMP signal-conversion domain: static two-state or dynamic three-state? Molecular Microbiology. 91, 853-857 (2014).

52. K. E. Swain, J. J. Falke, Structure of the Conserved HAMP Domain in an Intact, Membrane-Bound Chemoreceptor: A Disulfide Mapping Study. Biochemistry. 46, 1368413695 (2007).

53. M. V. Airola, K. J. Watts, A. M. Bilwes, B. R. Crane, Structure of Concatenated HAMP Domains Provides a Mechanism for Signal Transduction. Structure. 18, 436-448 (2010).

54. I. Gushchin, V. Gordeliy, S. Grudinin, Two Distinct States of the HAMP Domain from Sensory Rhodopsin Transducer Observed in Unbiased Molecular Dynamics Simulations. PLOS ONE. 8, e66917 (2013).

55. F. W. Studier, Protein production by auto-induction in high-density shaking cultures. Protein Expression and Purification. 41, 207-234 (2005).

56. I. Gushchin et al., Crystal structure of a light-driven sodium pump. Nat Struct Mol Biol. 22, 390-395 (2015). 
57. I. Gushchin et al., Structural insights into the proton pumping by unusual proteorhodopsin from nonmarine bacteria. PNAS. 110, 12631-12636 (2013).

58. D. Nurizzo et al., The ID23-1 structural biology beamline at the ESRF. Journal of Synchrotron Radiation. 13, 227-238 (2006).

59. P. Evans, Scaling and assessment of data quality. Acta Crystallographica Section D Biological Crystallography. 62, 72-82 (2005).

60. W. Kabsch, XDS. Acta Crystallographica Section D Biological Crystallography. 66, 125132 (2010).

61. A. Vagin, A. Teplyakov, Molecular replacement with MOLREP. Acta Crystallographica Section D Biological Crystallography. 66, 22-25 (2009).

62. G. M. Sheldrick, Experimental phasing with SHELXC/D/E: combining chain tracing with density modification. Acta Crystallogr D Biol Crystallogr. 66, 479-485 (2010).

63. T. Pape, T. R. Schneider, HKL2MAP: a graphical user interface for macromolecular phasing with SHELX programs. Journal of applied crystallography. 37, 843-844 (2004).

64. G. Langer, S. X. Cohen, V. S. Lamzin, A. Perrakis, Automated macromolecular model building for X-ray crystallography using ARP/wARP version 7. Nat. Protocols. 3, 11711179 (2008).

65. P. Emsley, K. Cowtan, Coot: model-building tools for molecular graphics. Acta Crystallographica Section D Biological Crystallography. 60, 2126-2132 (2004).

66. G. N. Murshudov et al., REFMAC5 for the refinement of macromolecular crystal structures. Acta Crystallographica Section D Biological Crystallography. 67, 355-367 (2011).

67. M. A. Lomize, I. D. Pogozheva, H. Joo, H. I. Mosberg, A. L. Lomize, OPM database and PPM web server: resources for positioning of proteins in membranes. Nucleic Acids Research. 40, D370-D376 (2012).

68. A. Hoffmann, and S. Grudinin, NOLB: Nonlinear rigid block normal-mode analysis method. J. Chem. Theory Comput. 13, 2123-2134 (2017). doi:10.1021/acs.jctc.7b00197. 


\section{Acknowledgments:}

Atomic coordinates and structure factors for the reported crystal structures have been deposited with the Protein Data Bank under the accession codes 5IJI, 5JEF and 5JEQ. The work was supported by the CEA(IBS) - HGF(FZJ) STC 5.1 specific agreement and the Russian Federal Target Program 14.587.21.0026 (RFMEFI58716X0026). The work used the platforms of the Grenoble Instruct Centre (ISBG; UMS 3518 CNRS-CEA-UJF-EMBL) with support from FRISBI (ANR-10-INSB-05-02) and GRAL (ANR-10-LABX-49-01) within the Grenoble Partnership for Structural Biology (PSB).

\section{Author contributions:}

I.G., A.I. and V.G. designed the study, A.I. expressed and purified the WT protein, I.G., P.B. and T.B. helped with expression and purification of the WT protein, A.Yu. expressed and purified the R50K mutant, I.G., I.M., V.P. and A.I. crystallized the protein, E.R. helped with crystallization, A.P. solved the structures, G. Bourenkov and G.L. helped with structure solution, I.G. and I.M. refined the structures, I.G. analyzed the structures, S.G., V.B., D.W. and G. Büldt helped with structure analysis, I.G and V.G. oversaw the study, analyzed the results and prepared the manuscript with contributions from all other coauthors.

\section{Supplementary Materials:}

21 Figures S1-S10

22 Table S1

23 Movies S1-S2 


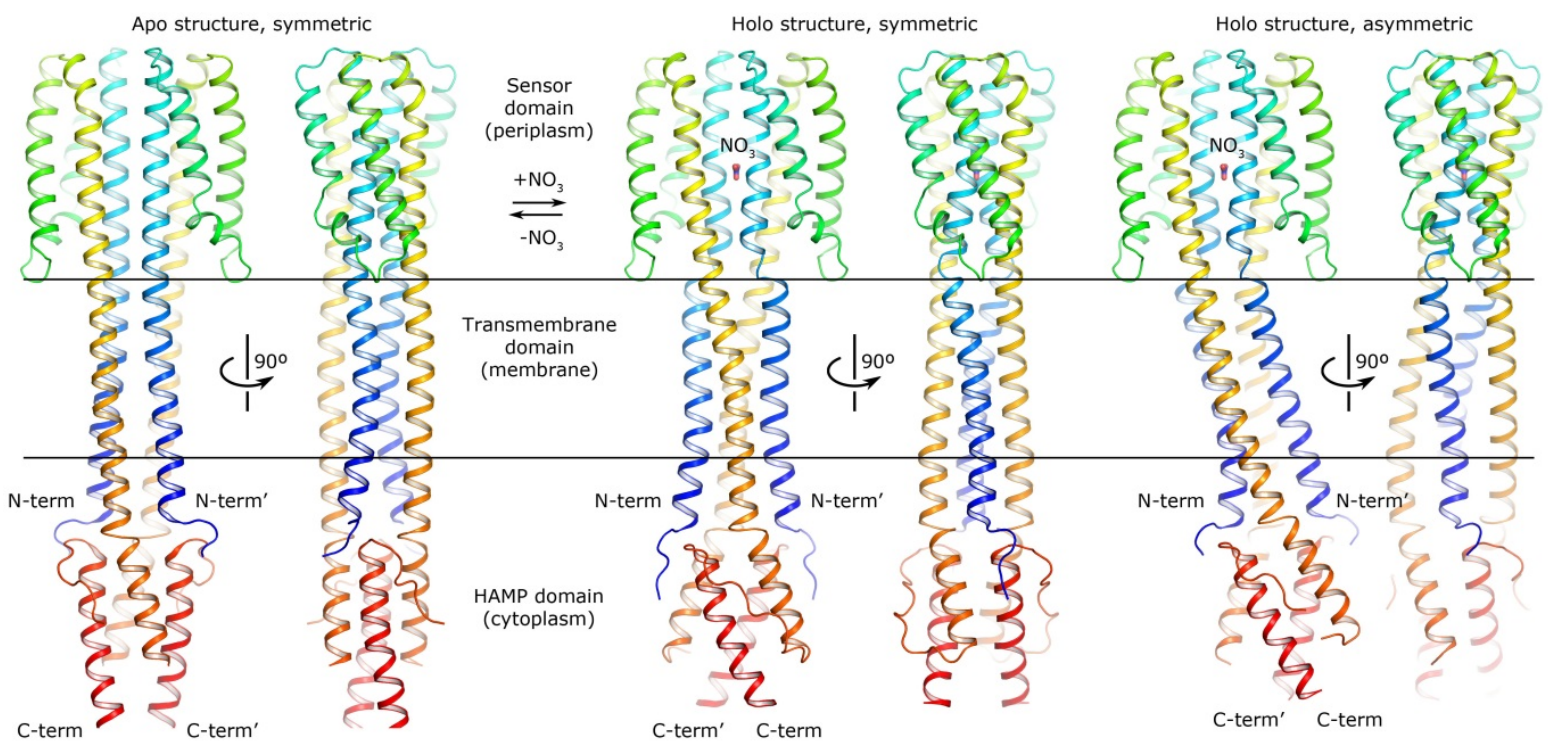

Figure 1. Overall structure of the crystallized NarQ fragment in apo and holo states. The protein is observed as a symmetric dimer in both the apo and holo-S states in the space groups $\mathrm{I} 2{ }_{1} 22_{1}$ and F222, respectively, and as an asymmetric dimer in holo-A state in the space group P2. Termini of the second protomer are denoted with primes. The crystallized fragment comprises the transmembrane domain (helices TM1 and TM2), the periplasmic sensor domain (helices H1-H4) with the nitrate ion binding at the dimerization interface, and the intracellular HAMP domain (helices AS1 and AS2). The structures are aligned by sensor domains. Hydrophobic membrane boundaries (black) were calculated using the OPM server (67) with the symmetric ligand-bound structure as a template. 


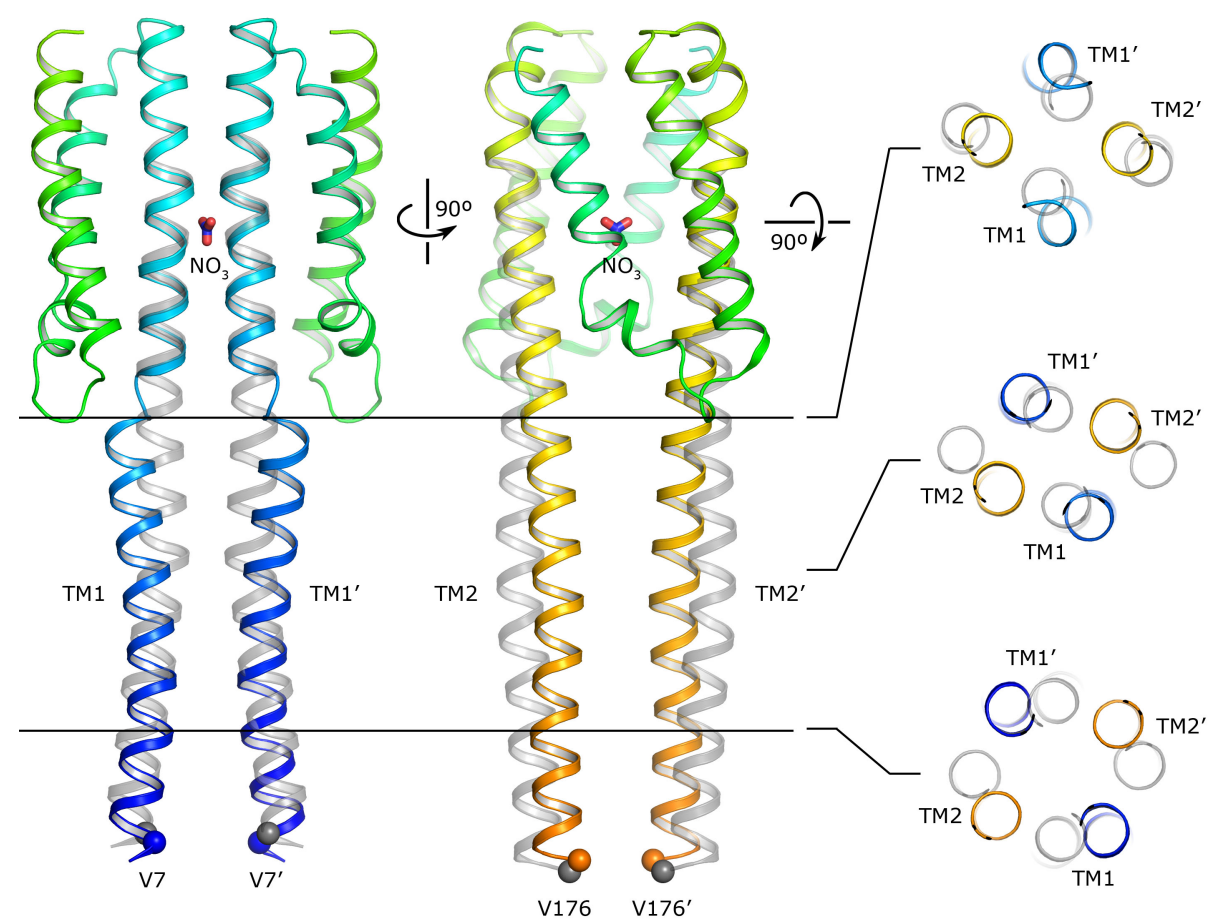

Figure 2. Signaling-associated conformational changes in the NarQ TM domain. Superposition of the apo state structure (grey) and symmetric holo state structure (colored) is shown. Left, changes in conformation of the helix TM1. Middle, changes in conformation of the helix TM2. Right, changes in the arrangement of the TM helices. $\mathrm{C}_{\alpha}$ atom positions of the residues from $\mathrm{CC}$ layer 9 are marked with the spheres to highlight the displacements in the direction perpendicular to the membrane. The structures are aligned by the sensor domains. 


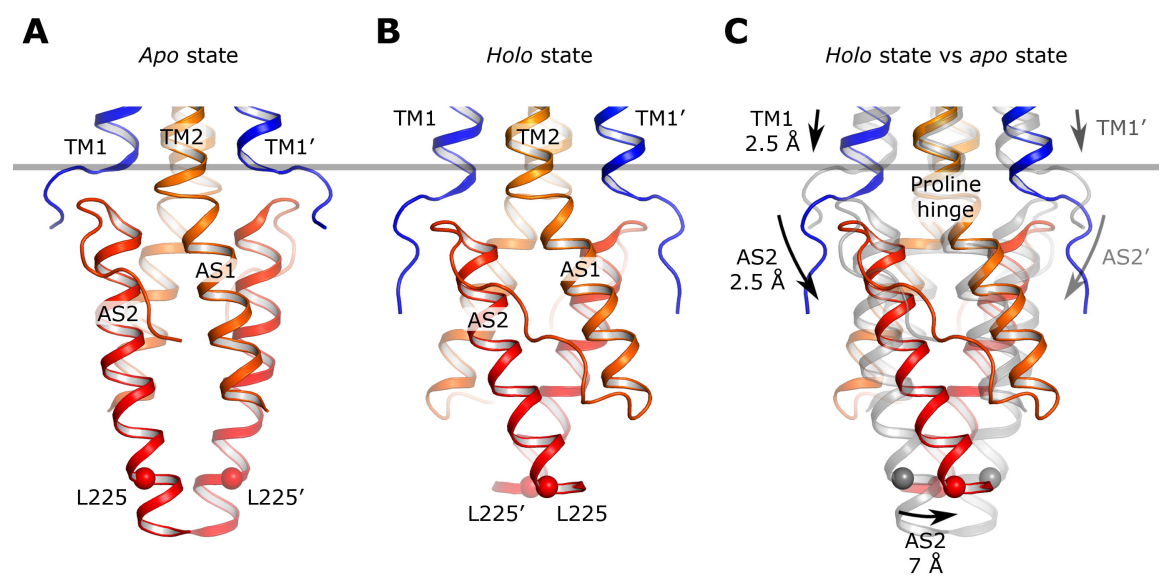

Figure 3. Details of the signal transduction from the TM domain to and through the HAMP domain. (A) inactive apo state. (B) active holo-S state. (C) superposition of the apo (grey) and holo-S (colored) states. A piston-like displacement of the cytoplasmic end of TM1 relative to TM2 and the TM2-AS1 hinge is transmitted to the membrane-proximal end of AS2 and results in lever-like rotations of the HAMP domain protomers around the hinges. Since the HAMP domain protomers move in opposite directions, the positions of membrane-distal ends of helices AS2 also change relative to each other. Positions of the Leu225 $\mathrm{C}_{\alpha}$ atom are marked with the spheres.

10 The grey bar shows the position of TM1 ends in the apo state structure. The domains are aligned 11 by the residues $175-177$. 


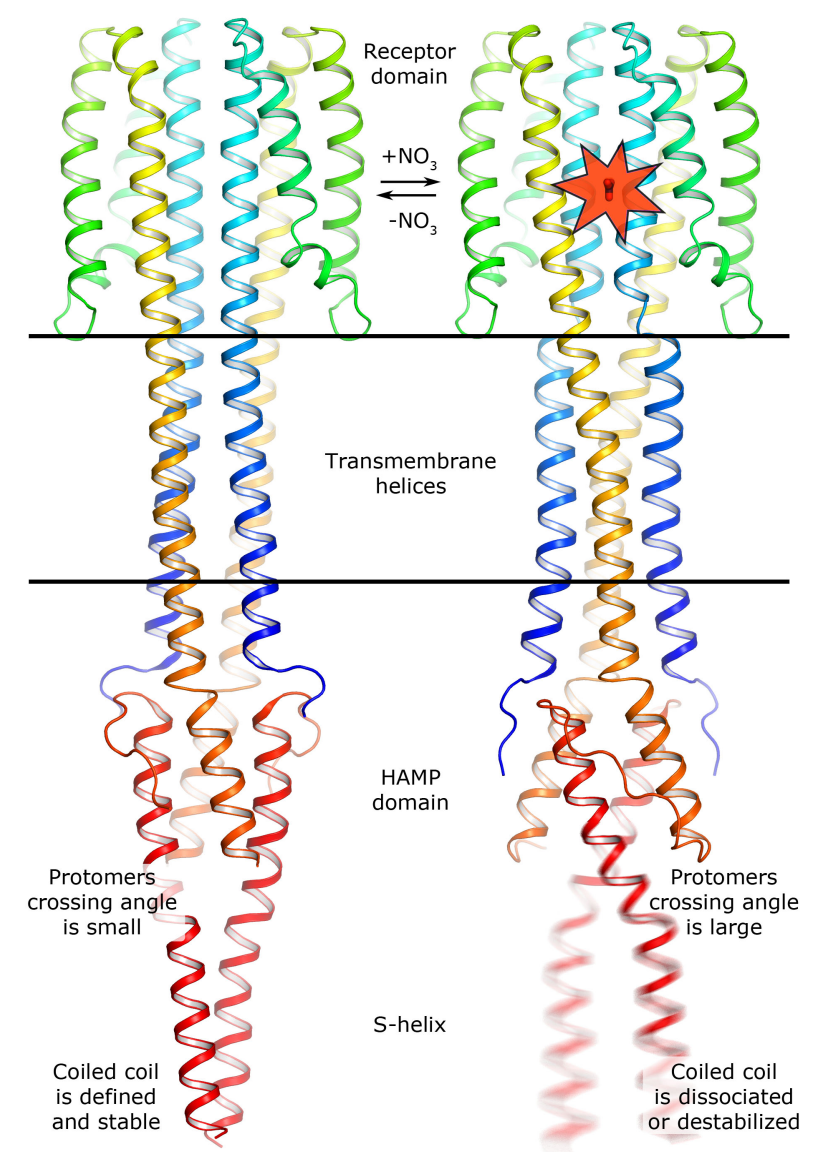

Figure 4. Mechanism of NarQ transmembrane signaling. Binding of the ligand results in a piston-like displacement towards the periplasm of the helices TM1 relative to TM2 and consequent lever-like conformational changes in the HAMP domain, which cause dissociation or destabilization of the signaling helix. 


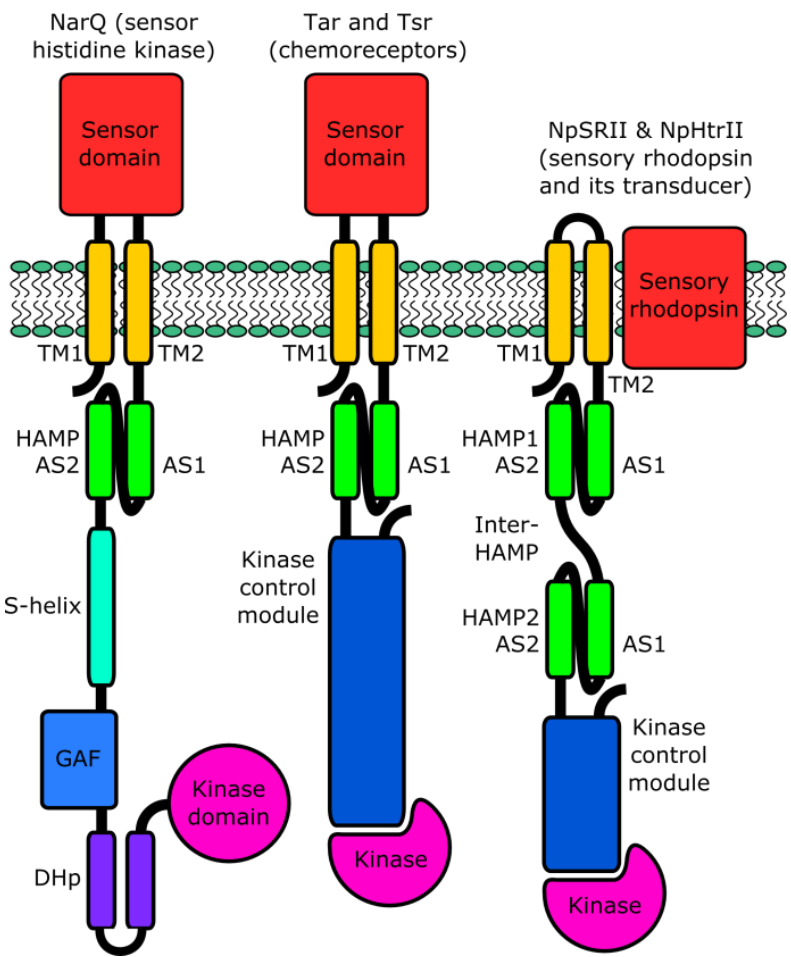

Figure S1. Domain architectures of representative microbial membrane sensor proteins. TM1 and TM2, transmembrane helices. AS1 and AS2, HAMP domain helices. NarQ is E. coli nitrate and nitrite sensor, Tsr is $E$. coli serine chemoreceptor, $N p$ SRII and $N p H$ trII are Natronomonas pharaonis sensory rhodopsin II and sensory rhodopsin transducer II. In chemo- and photoreceptor systems, the kinase is a separate protein. While only monomers are shown, all of the proteins are normally dimeric with chemo- and photoreceptor dimers forming higher-order oligomeric assemblies. 

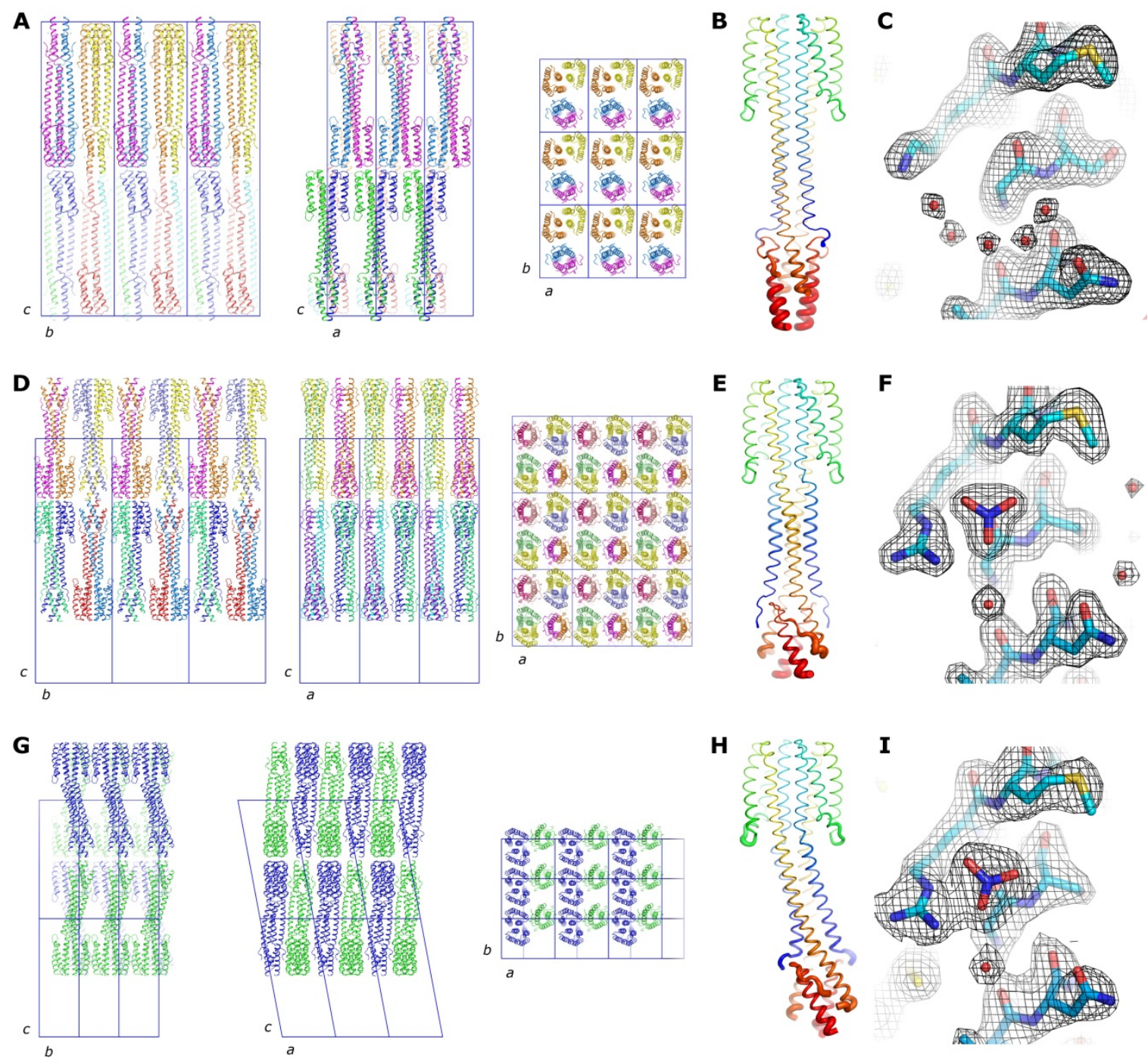

Figure S2. Crystallographic details. (A-C) Data for the space group I $2{ }_{1} 2_{1} 2_{1}$ (symmetric apo state structure). (D-F) Data for the space group F222 (symmetric holo state structure). (G-I) Data for the space group P2 (asymmetric holo state structure). (A, D, G) Crystal packing. The NarQ dimers pack in layers, as is typical for Type I membrane protein crystals, obtained through in meso crystallization. $(\mathbf{B}, \mathbf{E}, \mathbf{H})$ Relative distributions of the B-factor values. The scale in each drawing is different. In each space group, the membrane-distal parts of the HAMP domain are notably disordered. (C, F, I) Example of the electron density maps for the chain A residues around the ligand binding site. Weighted $2 \mathrm{~F}_{\mathrm{o}}-\mathrm{F}_{\mathrm{c}}$ electron density maps are contoured at the level of $1.5 \sigma$. 
A

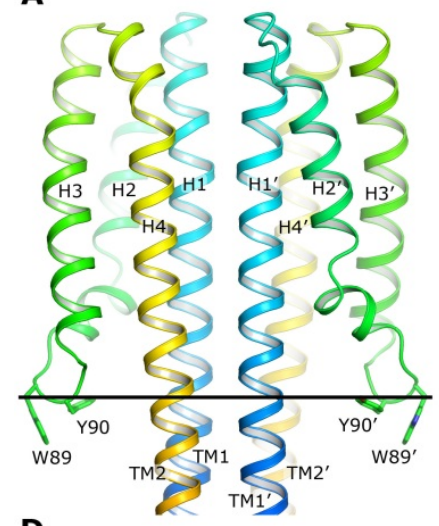

D

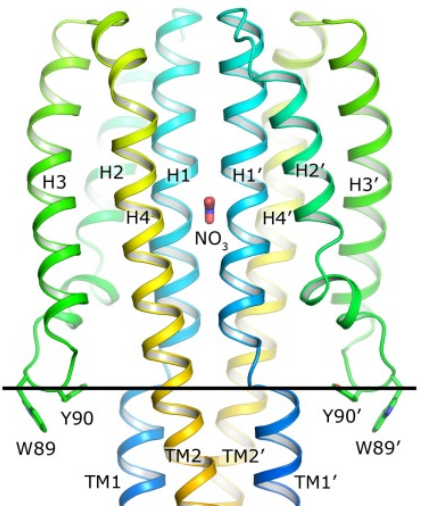

B

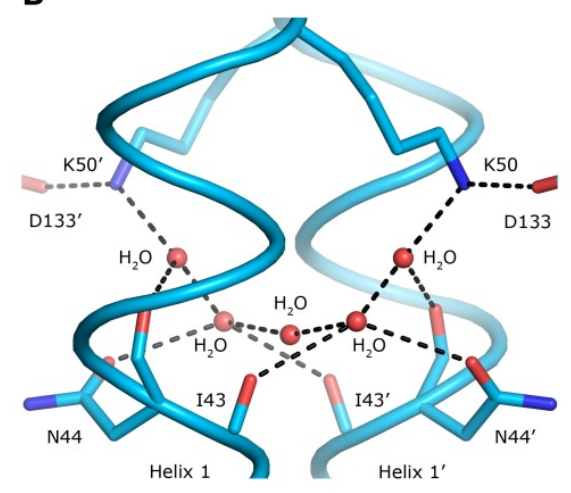

$\mathbf{E}$

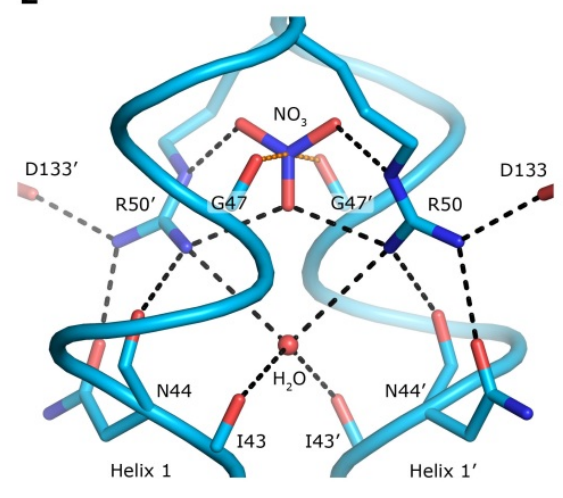

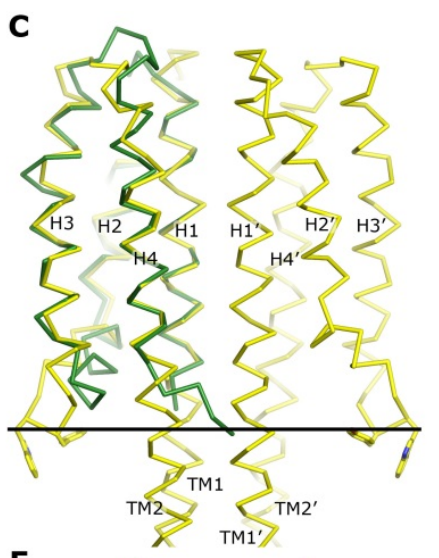

$\mathbf{F}$

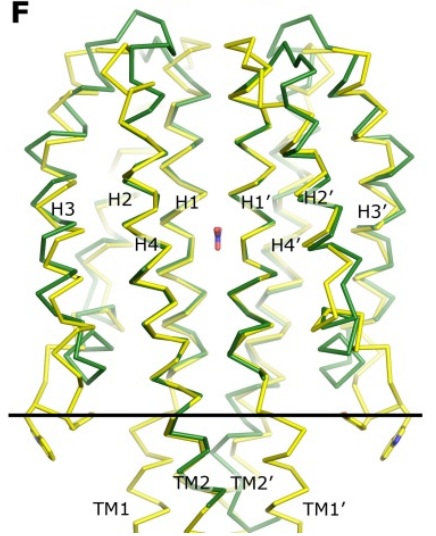

Figure S3. Structure of the NarQ periplasmic sensor domain. (A-C) Structure of the sensor domain in the apo state. The R50K mutant was used for structure determination. (A) Overall structure of the domain. The H2-H3 loop is anchored into the membrane with the residues Trp89 and Tyr90. (B) Ligand-binding site of apo NarQ. Three ordered water molecules are observed. (C) Comparison of the NarQ (yellow) and NarX (green, chain A from PDB: 3EZI (21)) sensor domains in the absence of ligands. Positions of the backbone atoms of the helices H1 and H4 are virtually indistinguishable. (D-F) Structure of the sensor domain in the holo state. (D) Overall structure of the domain. The ligand is bound at the dimerization interface between the helices $\mathrm{H} 1$ and H1'. The H2-H3 loop is anchored into the membrane with the residues Trp89 and Tyr90. (E) Nitrate-binding site of NarQ. The ion is bound by Arg50 side chains and Gly47 backbone oxygen atoms. Below the nitrate ion, a tetrahedrally coordinated water molecule is observed. Potential hydrogen bonds are shown as black dashed lines. The interaction of $\mathrm{NO}_{3}{ }^{-}$partially positively charged nitrogen atom with the Gly47 partially negatively charged backbone oxygen atom is shown with orange dashing. (F) Comparison of the ligand-bound NarQ (yellow) and NarX (green, PDB: 3EZH (21)) sensor domains. Positions of the backbone atoms of the helices $\mathrm{H} 1$ and $\mathrm{H} 4$ are virtually indistinguishable. Positions of the TM2 backbone atoms present in the NarX construct used for crystallization(21) are also identical to those of NarQ. Positions of the NarX TM2' backbone atoms are different, possibly due to the absence of corresponding TM1 and TM1' residues that would stabilize the correct conformation. Note that NarX position relative to the membrane boundaries probably differs from that of NarQ (not shown). 

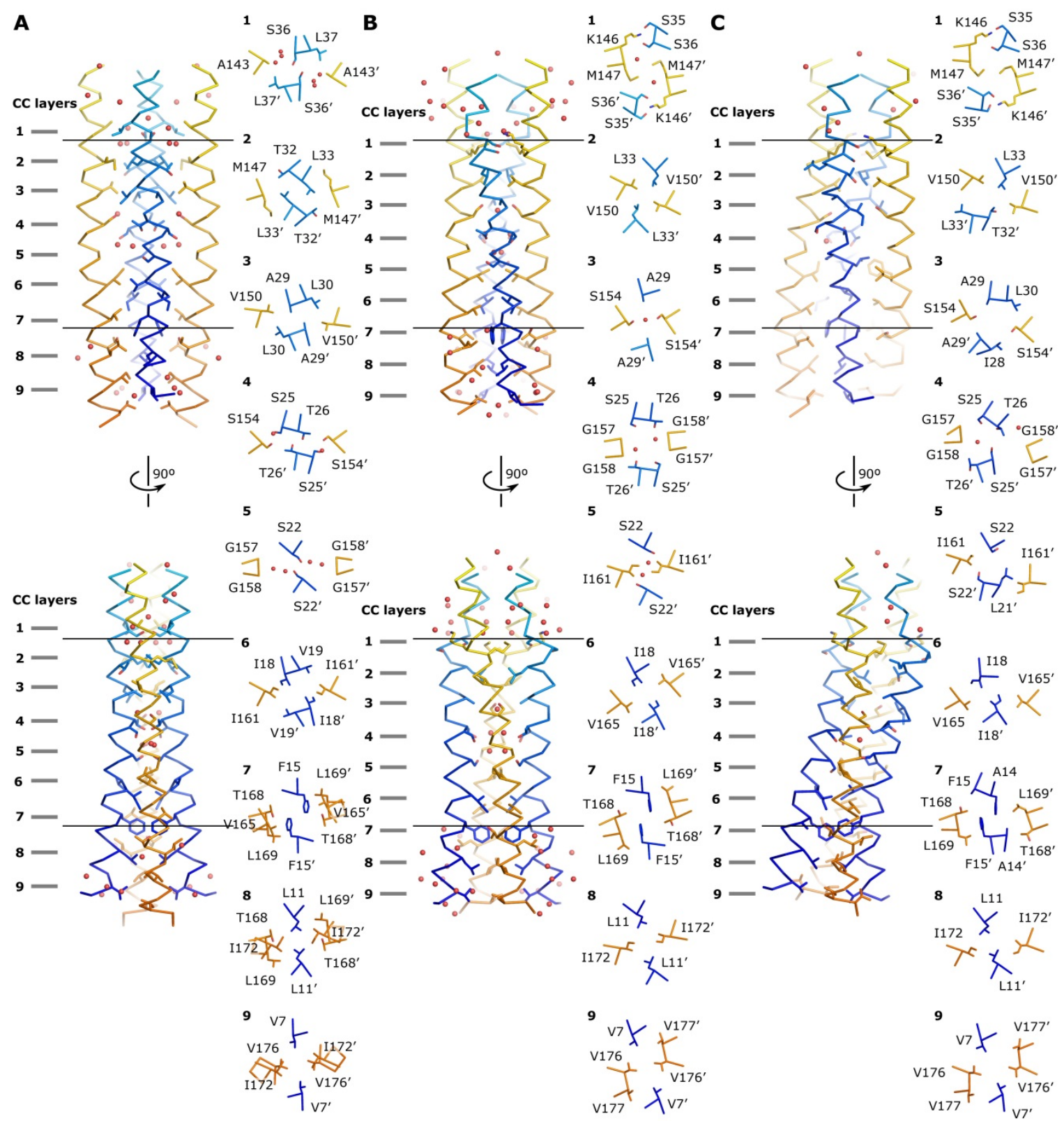

Figure S4. Structure of the NarQ transmembrane domain. TM1 is in blue, and TM2 is in yellow. (A) Apo state. (B) Symmetric holo state. (C) Asymmetric holo state. In each panel, overall structure (left) and side chain packings in the $9 \mathrm{CC}$ layers (right, view from the periplasm) are shown. In each structure, ordered water molecules are observed in or around CC layer 4. 


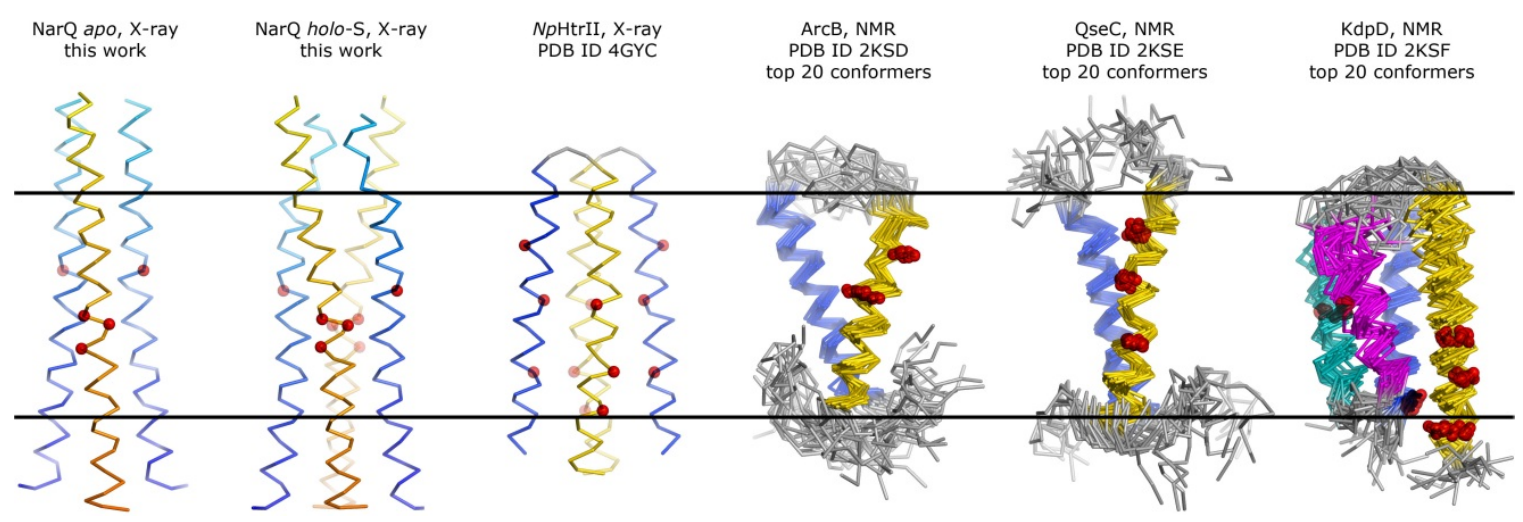

Figure S5. Comparison of the experimentally determined structures of TCS TM domains. In each case, the Nterminal $\alpha$-helix TM1 is shown in blue and the C-terminal one is shown in yellow. Glycine and proline residues of the TM helices are shown with red spheres. There are generally more glycine and proline residues in the last TM helix of the histidine kinases. The scale is the same for all of the proteins. X-ray structure of $N p H \operatorname{trII}(28)$ is aligned to ligand-bound NarQ structure using the positions of the HAMP-domain proximal residues that are similar, opposite to the positions of the residues in the middle of the membrane and at the periplasmic side. Since there is no sequence similarity between the TM domains, NMR structures of ArcB, QseC and $\mathrm{KdpD}(15)$ are aligned to the NarQ structure so that their helices are roughly at the same positions relative to the membrane. Structural comparison with $\mathrm{ArcB}, \mathrm{QseC}$ and $\mathrm{KdpD}$ is hampered since the structures are monomeric and information about the dimerization interfaces is lacking (15). It is not clear whether the $\mathrm{ArcB}$ and $\mathrm{QseC}$ fragments are in the correct physiological state. In the KdpD structure, presence of the additional TM helices (TM2 and TM3, cyan and magenta) probably ensures correct folding of the TM domain and proper orientations of the N-terminal and Cterminal helices. 
A

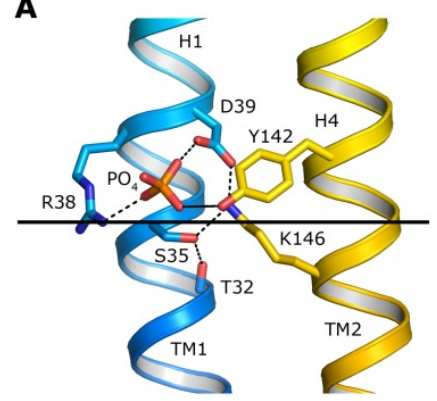

D

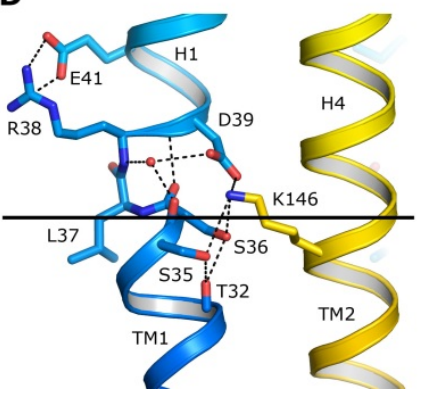

B

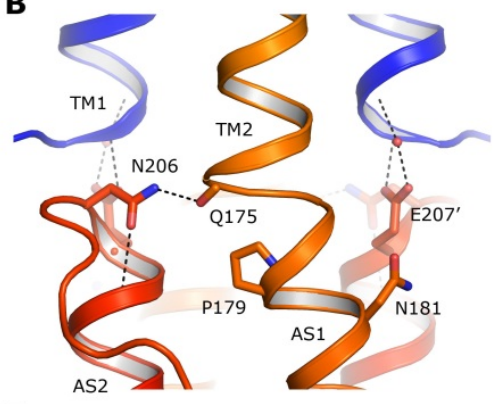

E

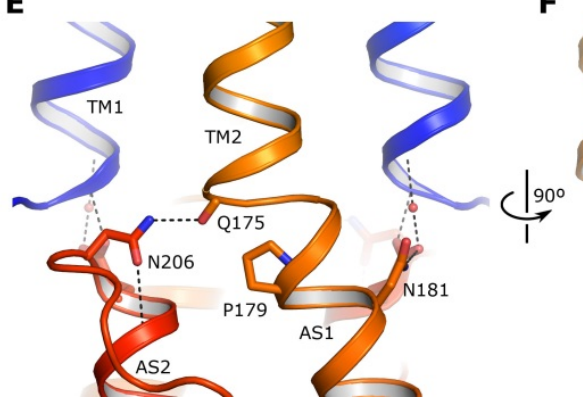

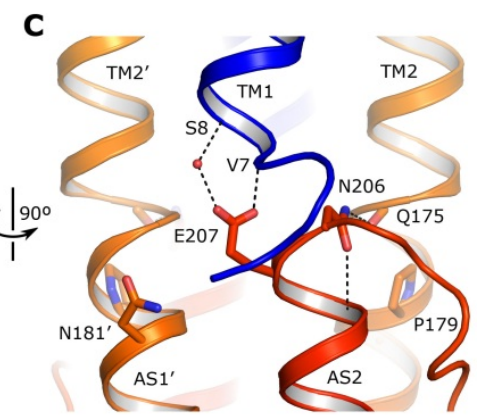

$\mathbf{F}$

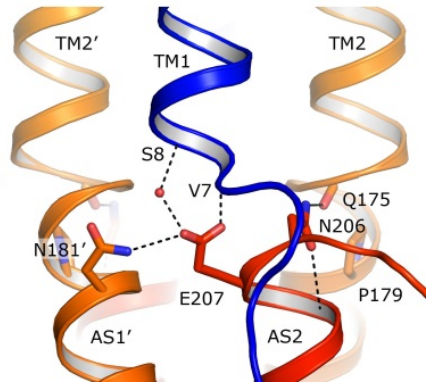

Figure S6. Structure of the junctions between the NarQ TM helices and adjacent domains. (A-C) Apo state structure. (A) Connections between the TM helices and the sensor domain. Both TM1-H1 and TM2-H4 transitions maintain the helical structure. A phosphate anion is bound close to the hydrophobic membrane boundary. Putative hydrogen bonds are shown with the black dashes. (B) and (C) Connections between the TM helices and the HAMP domain. (B) Structure of the proline kink in the TM2-AS1 junction. (C) Structure of the TM1-AS2 link. Cytoplasmic terminus of TM1 is connected to the membrane-proximal end of AS2 via two hydrogen bonds. One bond is direct (Val7-Glu207) and the other is mediated by a conserved water molecule (Ser8-Glu207). (D-F) Holo-S symmetric state structure. (D) Connections between the TM helices and the sensor domain. There is a break in the helical structure at the TM1-H1 junction, while the H4-TM2 transition is continuous. The break is stabilized by a water molecule and several hydrogen bonds (black dashes). (E) and (F) Connections between the TM helices and the HAMP domain. (E) Structure of the proline kink in the TM2-AS1 junction. There is a hydrogen bond between Asn206 and Gln175 backbone carbonyl oxygen atom that connects the cytoplasmic end of TM2 and membraneproximal end of AS2. (F) Structure of the TM1-AS2 link. The cytoplasmic terminus of TM1 is connected to the membrane-proximal end of AS2 via two hydrogen bonds. One bond is direct (Val7-Glu207) and the other is mediated by a conserved water molecule (Ser8-Glu207). Glu207 side chain conformation is also stabilized by a hydrogen bond with Asn181' of the adjacent helix AS1'. 
A
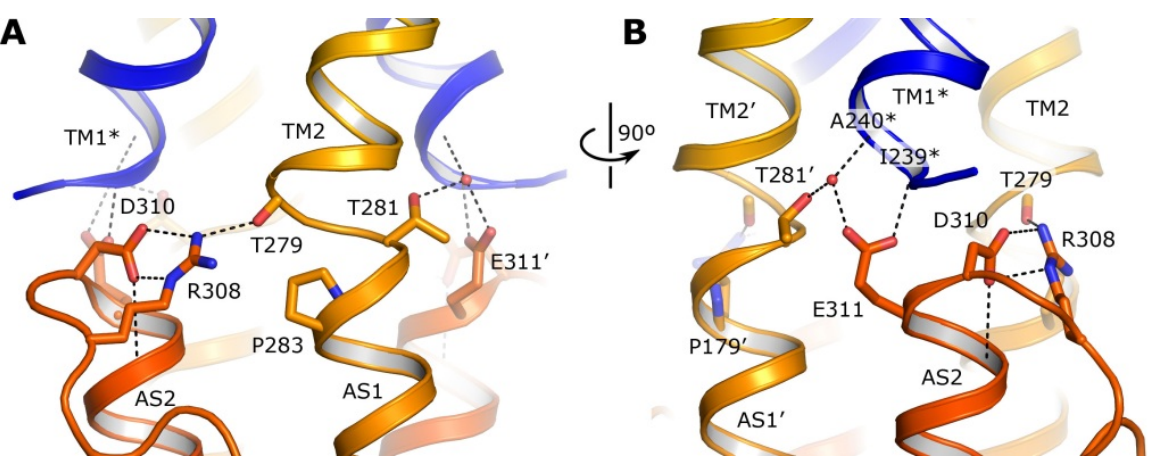

Figure S7. Structure of the junctions between the TM helices and HAMP domain in Af1503 (PDB: 4CQ4 (30)). (A) Structure of the proline kink in the TM2-AS1 junction. (B) Structure of the TM1*-AS2 link. Cytoplasmic terminus of the helix TM1* is connected to the membrane-proximal end of AS2 via two hydrogen bonds. One bond is direct (Ile239*-Glu311) and the other is mediated by a water molecule (Ala240*-Glu311). The water molecule is also stabilized by a hydrogen bond with the side chain of Thr181' of the adjacent helix AS1'. Notation TM1* is chosen to emphasize that the structure corresponds to unnatural antiparallel tetramer of the construct comprising the mutated TM2 helix and the HAMP domain of the Af1503 protein (30). Consequently, positions of TM1 in the correctly folded full-length Af1503 can differ from those presented. 


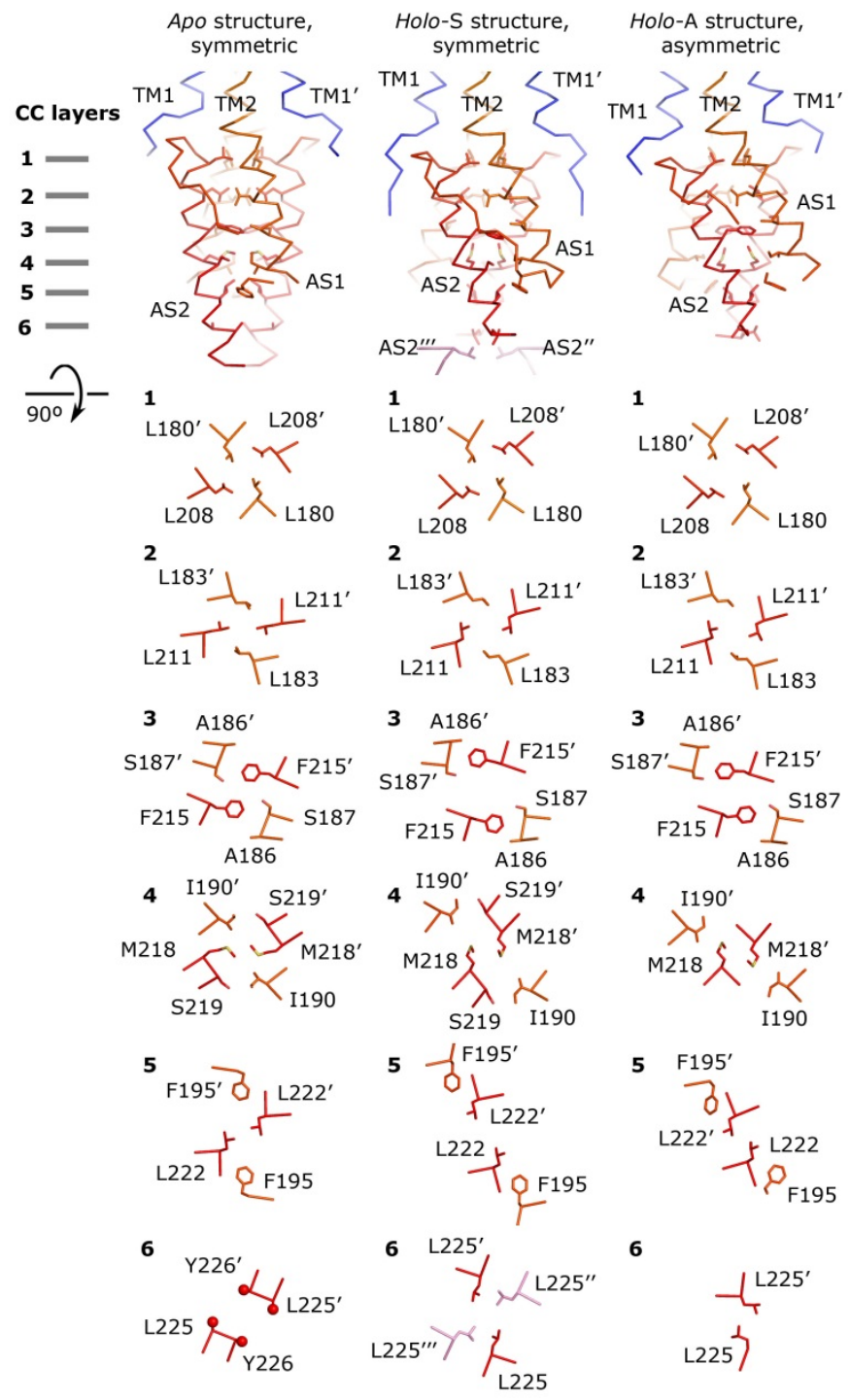

Figure S8. Structure of the NarQ HAMP domain in apo, symmetric holo and asymmetric holo states. Structures are aligned by the residues of the TM2-AS1 junction. In the apo state structure, side chains of the residues Leu225 and Tyr226 are not resolved and consequently are marked with spheres. In the symmetric holo state structure, AS2 helices of symmetry-related molecules are marked AS2" and AS2"', and only one of two alternative side chain conformations of Leu225 is shown in each case. Note that Phe195 of layer 5 resides on the linker and not on AS1 or AS2, and that not all of the linker residues are resolved in the apo and asymmetric holo state structures. 


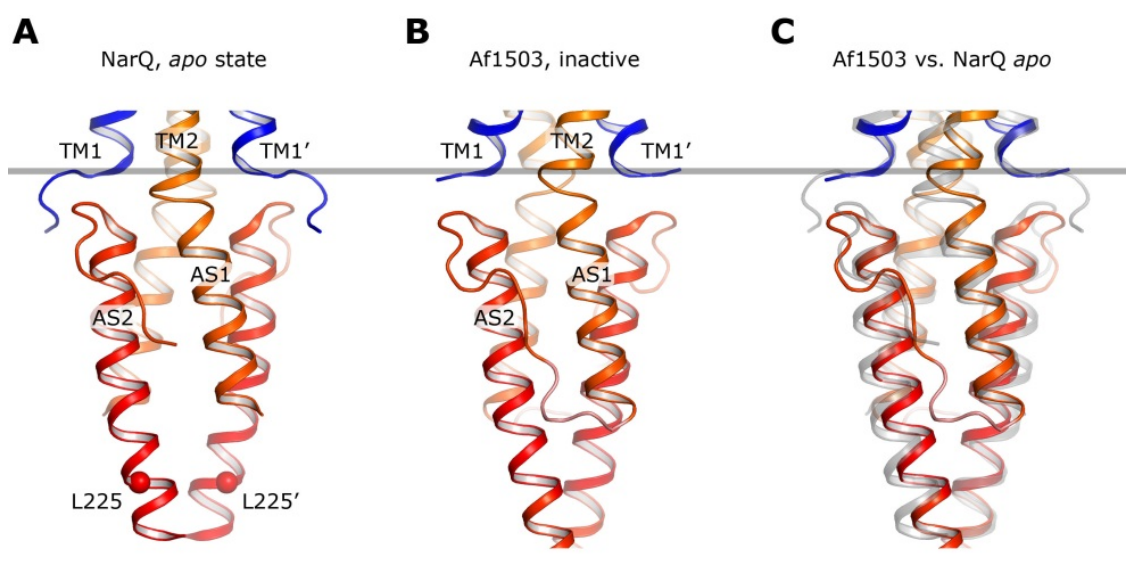

Figure S9. Comparison of the NarQ apo state HAMP domain and Af1503 soluble mutant HAMP domain. (A) Structure of the HAMP domain in the apo state of NarQ. (B) Structure of the HAMP domain in the soluble mutant of Af1503 receptor (PDB: 4CQ4) (30). (C) Superposition of the apo-state NarQ HAMP domain (grey) and Af1503 HAMP domain (colored). The HAMP domain structures are similar. The grey line shows the position of TM1 ends in the apo state NarQ structure. 


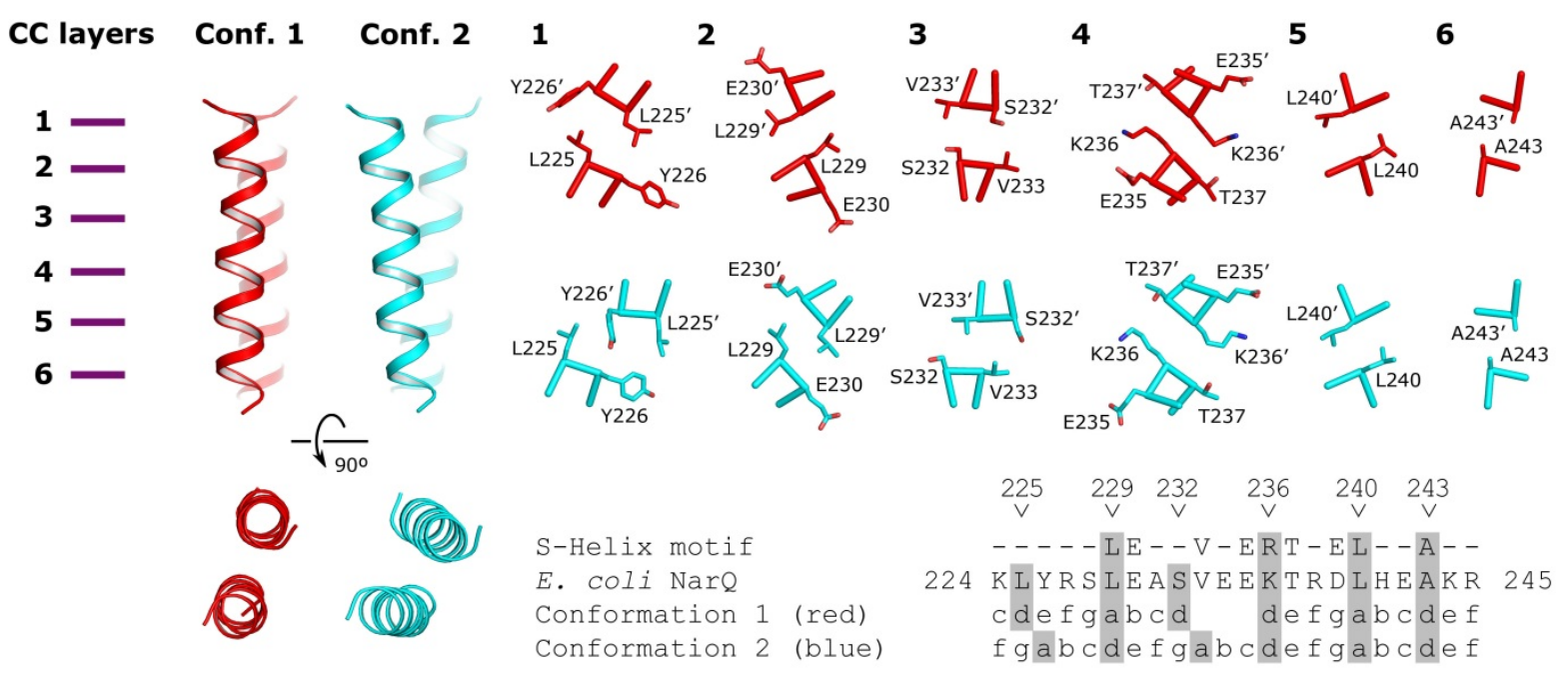

Figure S10. Coiled coil models of NarQ signaling helix domain. Two conformations are possible. Conformation 1 is shown in red and conformation 2 is shown in blue. The packing in the layers 1-3 is different, while the packing in the layers 4-6 is similar. The phase stutter is possible in the region of the residues 232-236. Residues Glu235, Lys236, Thr237 belong to the characteristic conserved signaling helix motif (32). 
4

5

6

7

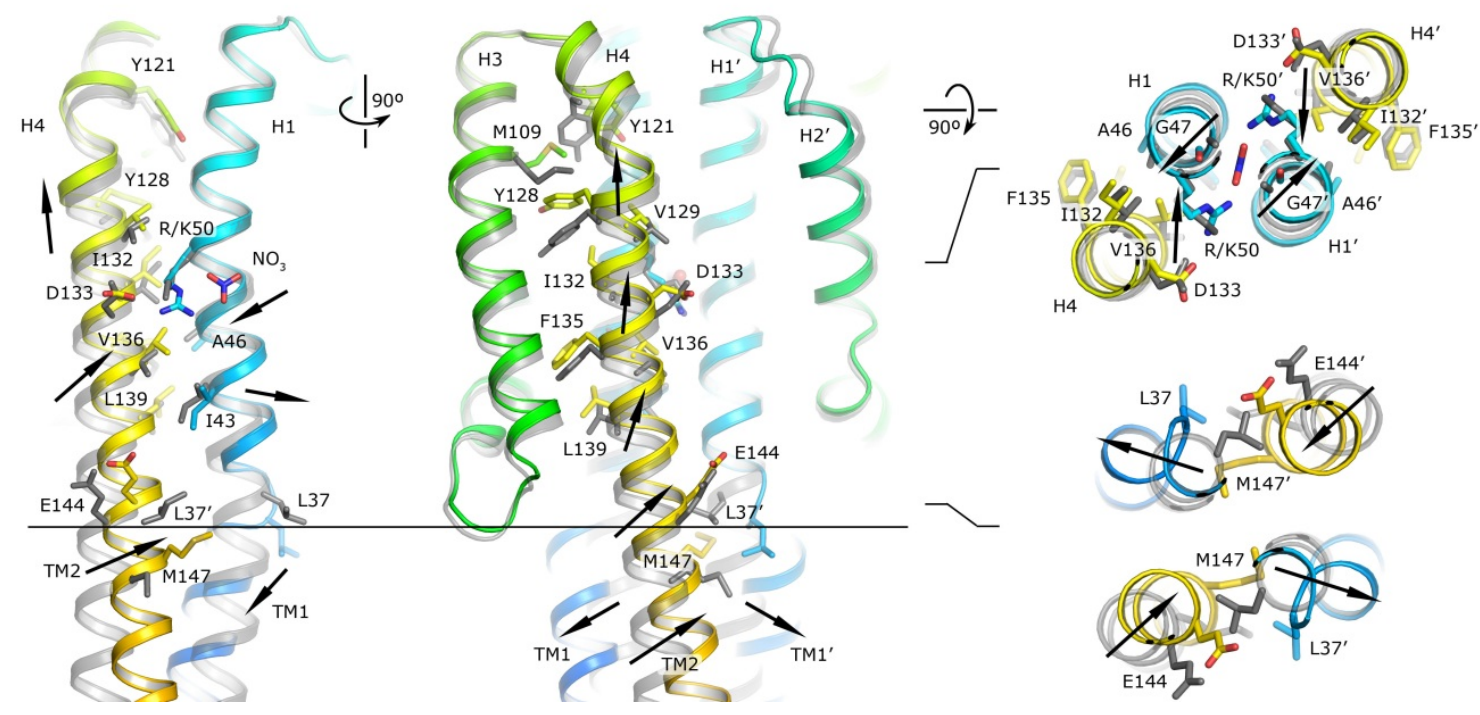

Figure S11. Signaling-associated conformational changes in the NarQ sensor domain. Superposition of the apo state structure (grey) and symmetric holo state structure (colored) is shown. The black arrows indicate the directions of the displacements of NarQ elements upon binding of the ligand (not drawn to scale). The structures are aligned using the residues 48-53 (membrane-distal part of the helix H1). 
Table S1. Data collection, phasing and refinement statistics

\begin{tabular}{|c|c|c|c|c|}
\hline & Apo & Holo F222 NaI & Holo F222 Native & Holo P2 \\
\hline \multicolumn{5}{|l|}{ Data collection } \\
\hline Space group & $\mathrm{I} 2{ }_{1} 2_{1} 2_{1}$ & F222 & F222 & $\mathrm{P} 2$ \\
\hline \multicolumn{5}{|l|}{ Cell dimensions } \\
\hline$a, b, c(\AA)$ & 39.59, & 57.17, & 57.58 & 53.46 , \\
\hline & 59.37 & 73.48 & 73.87 & 39.87 \\
\hline & 239.58 & 236.45 & 235.56 & 120.36 \\
\hline$\alpha, \beta, \gamma\left({ }^{\circ}\right)$ & $90,90,90$ & $90,90,90$ & $90,90,90$ & $90,100.62,90$ \\
\hline Wavelength $(\AA)$ & 0.972 & 1.85 & 0.972 & 0.972 \\
\hline \multirow[t]{2}{*}{ Resolution $(\AA)$} & $47.64-1.90$ & $60-2.7$ & 39.4-1.94 & $52.55-2.42$ \\
\hline & $(1.94-1.90)^{*}$ & $(2.9-2.7)$ & $(1.99-1.94)$ & $(2.51-2.42)$ \\
\hline$R_{\text {merge }}(\%)$ & $7.5(156.4)$ & $17.1(74.4)$ & $8.3(98.9)$ & $9.4(66.6)$ \\
\hline$I / \sigma I$ & $11.8(1.2)$ & $21.7(3.4)$ & $14.5(2.0)$ & $7.6(1.8)$ \\
\hline$C C_{1 / 2}(\%)$ & $99.8(80.2)$ & $100(93.9)$ & 99.9 (77.9) & $99.5(67.2)$ \\
\hline$C C_{\text {anom }}(\%)$ & & 79 (14) & & \\
\hline Completeness (\%) & $99.9(99.9)$ & $99.9(99.8)$ & $98.9(98.1)$ & $96.6(96.6)$ \\
\hline Unique reflections & $22891(1426)$ & $13242(2584)$ & $18674(1221)$ & $18809(1732)$ \\
\hline Redundancy & $6.4(6.6)$ & $43.0(13.3)$ & $5.5(5.8)$ & $2.8(2.9)$ \\
\hline \multicolumn{5}{|l|}{ Refinement } \\
\hline Resolution ( $\AA$ ) & $47.64-1.90$ & & 39.4-1.94 & $52.55-2.42$ \\
\hline No. reflections & 21742 & & 17728 & 17932 \\
\hline$R_{\text {work } /} R_{\text {free }}(\%)$ & $23.2 / 28.5$ & & $19.0 / 25.4$ & $21.8 / 28.6$ \\
\hline \multicolumn{5}{|l|}{ No. atoms } \\
\hline Protein & 1763 & & 1849 & 3388 \\
\hline Ligand & 0 & & 4 & 4 \\
\hline Water and ions & 119 & & 172 & 62 \\
\hline Lipids & 0 & & 34 & 6 \\
\hline \multicolumn{5}{|l|}{ B-factors } \\
\hline Protein & 51.6 & & 34.3 & 52.1 \\
\hline Ligand & - & & 27.7 & 28.2 \\
\hline Water and ions & 61.5 & & 42.8 & 41.0 \\
\hline Lipids & - & & 57.0 & 62.3 \\
\hline \multicolumn{5}{|l|}{ R.m.s deviations } \\
\hline Bond lengths $(\AA)$ & 0.007 & & 0.008 & 0.006 \\
\hline Bond angles $\left({ }^{\circ}\right)$ & 1.0 & & 1.1 & 0.9 \\
\hline \multicolumn{5}{|c|}{ Ramachandran analysis } \\
\hline Favored (\%) & 97 & & 98 & 97 \\
\hline Allowed (\%) & 3 & & 2 & 3 \\
\hline Outliers (\%) & 0 & & 0 & 0 \\
\hline
\end{tabular}

*Highest resolution shell is shown in parenthesis. 
1 Movie S1. Mechanism of transmembrane signaling by sensor histidine kinase NarQ.

3 Movie S2. Comparison of symmetric and asymmetric ligand-bound NarQ structures.

4 